\title{
Estudio gravimétrico del cuadrángulo de Tegucigalpa, Valle del Zamorano, Valle de San Juan de Flores y Morocelí
}

\author{
Carlos A. Tenorio Moncada*, Elizabeth Espinoza Canales
}

\section{RESUMEN}

Entre el 4 de julio y el 17 de agosto del 2007, realizó una campaña de gravimetría con el propósito de recolectar datos de gravedad relativa en las regiones del cuadrángulo de Tegucigalpa, Valle del Zamorano y Valle de San Juan de Flores y Morocelí. En dicha campaña se establecieron 67 estaciones, con las cuales se formó una red gravimétrica local con 24 circuitos, interconectados mediante una estación base que forma parte de la red regional establecida por NOA-NGS (2001). A lo largo de toda la zona de influencia encontramos fuertes anomalías negativas de Bouguer, entre -57 y - 79 mGals, consistentes con los valores regionales reportados anteriormente por Bowin (1978) y Weyl (1980) asociadas a la compensación isostática en la zona central de Honduras.

En los valles de San Juan de Flores (Cantarranas), Morocelí y El Zamorano, definimos niveles de gravedad regional adecuados para explorar la topografía interna del basamento y las profundidades del aluvión a lo largo de perfiles selectos. Utilizando el programa GRAVMAG (Burger et al, 2006) que implementa el método de Talwani et al, (1959) para calcular gravedad residual, construimos modelos del cuerpo de aluvión en dos dimensiones consistentes con la geología y gravedad residual observada, asumiendo un contraste de densidad de $-0.4 \mathrm{~g} / \mathrm{cm}^{3}$. Los fondos más irregulares y profundos se observaron en el valle de Morocelí, para un perfil longitudinal de $37 \mathrm{~km}$ en el cual las profundidades oscilan entre 1000, 400 y 1,400 m.

Los Valles de San Juan de Flores y Zamorano, por el contrario, exhiben una topografía interna suave y profundidades máximas de 130 y $350 \mathrm{~m}$., respectivamente. Para la zona metropolitana de la ciudad de Tegucigalpa elaboramos un mapa de contorno de anomalías de Bouguer y observamos una fuerte correlación con la geología local, con un máximo alrededor de -60 mgals asociado a la formación Río Chiquito (Krc) del grupo de Valle de Angeles, y valores bajos, hasta -80 mGals, en las zonas de tobas y sedimentos volcánicos del Grupo

*Universidad Nacional Autónoma de Honduras. Facultad de Ciencias. Escuela de Física 
Padre Miguel. Esto significa que es posible definir curvas de gravedad regional asociada a la zona de contacto entre estos dos grupos, una variable de gran importancia geotécnica para la industria local de la construcción. Nuestra exploración preliminar del campo de gravedad residual en Tegucigalpa sugiere un nivel de gravedad regional de - $67 \mathrm{mG}$ als para hacer correcciones de isostasia en futuros trabajos de exploración gravimétrica en esta ciudad.

\section{Palabras claves: Gravimetría, GPS, anomalías de Bouguer, anomalías residuales, perfil gravimétrico.}

\section{ABSTRACT}

Between July 4 and August 17, 2007, conducted a campaign of gravimetry for the purpose of gathering data relative gravity of the quadrangle areas of Tegucigalpa, Zamorano Valley and Valle de San Juan de Flores and Morocelí. In that campaign they were established 67 stations, with which it formed a local gravimetric network with 24 circuits, interconnected by a base station that is part of the regional network established by NOA-NGS (2001). Throughout the whole area of ??nfluence strong negative Bouguer anomalies were found, between -57 and -79 mGals, consistent with previously reported regional values Bowin (1978) and Weyl (1980) associated with isostatic compensation in the central zone of Honduras.

In the valleys of San Juan de Flores (Cantarranas) Morocelí and El Zamorano, appropriate regional severity levels were defined to explore the internal topography of the basement and the depth of alluvium along selected profiles. Using the program GRAVMAG (Burger et al, 2006) that implements the method of Talwani et al, (1959) for calculating residual gravity, we build models of the body of alluvium in two dimensions consistent with the observed residual gravity geology and assuming a contrast $-0.4 \mathrm{~g} / \mathrm{cm} 3$ density. More irregular funds were seen deep in the valley of Morocelí, for a longitudinal profile of $37 \mathrm{~km}$ where the depths range from 1000, 400 and $1,400 \mathrm{~m}$.

The valleys of San Juan de Flores and Zamorano, however, exhibit a smooth internal topography and maximum depths of 130 and $350 \mathrm{~m}$, respectively. For the metropolitan area of Tegucigalpa developed a contour map of Bouguer anomalies and observed a strong correlation with local geology, with a maximum around -60 mgals associated with the formation Rio Chiquito $(\mathrm{Krc})$ Valley Group Angeles, and low values, down to $-80 \mathrm{mG}$ als in areas of volcanic tuffs and sediments of the Padre Miguel Group. This means it is possible to define regional gravity curves associated with the contact zone between these two groups, one very important variable for the 
local industry geotechnical construction. Our preliminary exploration of the residual gravity field in Tegucigalpa suggests a regional severity level to $-67 \mathrm{mGals}$ isostasy corrections in future gravimetric exploration work in this city.

Keywords: Gravity, GPS, Bouguer anomalies, anomalies residual gravity profile. 


\section{INTRODUCCIÓN}

La gravimetría como método de exploración geofísica investiga las variaciones en la densidad de los materiales subyacentes en la corteza terrestre. El campo gravitacional de la Tierra tiene un valor promedio de $980 \mathrm{~cm} / \mathrm{s}^{2}$ (gals) debido a la masa total del planeta, con un rango de variación de aproximadamente 5 gals, ó $0.5 \%$ de $g$, entre los polos y el ecuador debido a la forma elipsoidal de la Tierra (Telford et al, 1987). De este valor, la mayor contribución proviene de la masa del núcleo y del manto. Solamente $0.3 \%$ del valor de $g$ se debe a materiales contenidos en la corteza y de esta fracción apenas un 15\% (0.05g) corresponde a los $5 \mathrm{~km}$ de roca más superficiales (Burger et al., 2006). Esta región suele ser el principal objeto de las técnicas de exploración geofísica por contener los cuerpos minerales de valor comercial y estructuras geológicas (fallas, grábenes, cuencas sedimentarias) que son de interés en estudios de exploración y riesgo.

Pocos estudios de gravimetría han sido emprendidos en Honduras a nivel local. En los años 50 y principios de los 60, la Universidad de Wisconsin estableció en Centro América estaciones de gravedad relativa con empleo de gravímetros Worden con precisión de \pm 0.3 mGals (N.O.A.A, 2001). A nivel regional, Weyl (1980) menciona que un conjunto de datos gravimétricos de América Central, hasta entonces inaccesibles y dispersos, fueron recopilados y ordenados por Bowin (1976) quien los interpreta a la luz de la teoría de la Tectónica de Placas. Para Honduras este estudio reporta fuertes anomalías negativas de Bouguer en las cordilleras montañosas, a diferencia de las zonas oceánicas de la cadena de volcanes del Pacífico con valores positivos. Estudios de gravedad relativa en zonas específicas fueron llevados a cabo por Zúñiga (1975) en el Valle de Sula y por Ad-Zadjali et al., (1996) en el Valle de Comayagua. El primero reporta profundidades máximas de 2000 m del basamento metamórfico en el norte del valle de Sula y el segundo ofrece un valor preliminar de $2700 \mathrm{~m}$ para la profundidad máxima del Valle de Comayagua. Posteriormente, gracias a la perturbación de la topografía causada por el Huracán Mitch en 1998, y como parte del Programa de Restauración patrocinado por el Servicio Geodésico Nacional de los E.E.U.U., se estableció una red de estaciones de gravedad absoluta en Centro América con una precisión de \pm 2 ì Gals. Dichas estaciones pueden usarse como estaciones de referencia para estudios gravimétricos locales. En Honduras se efectuaron mediciones precisas de $g$ en Tegucigalpa, San Lorenzo, Catacamas, Santa Rosa de Copán y San Pedro Sula . Véase la Tabla 1. (N.O.A.A., 2001).

Realizamos una campaña de gravimetría durante los meses de Junio, Julio y parte de Agosto de 2007 utilizando un gravímetro de la casa Lacoste \& Romberg, 
GRAVITONEG. Recolectamos un total de 188 datos gravimétricos, correspondiente a 67 estaciones, cubriendo una zona de influencia de 1,300 $\mathrm{km}^{2}$, incluyendo los datos de estaciones repetidas para realizar correcciones de mareas gravitacionales lunares y derivas del instrumento. Para este informe seleccionamos los datos asociados a las principales formaciones geológicas descritas en el mapa geológico de Tegucigalpa (Rodgers, et al, 1990) y la carta geológica del cuadrángulo de Morocelí (Markey, 1993), y el Valle del Zamorano.

Tabla 1: Localización y valores absolutos gravedad, de las estaciones de Honduras que forman parte de la Red Regional (N.O.A.A., 2001).

\begin{tabular}{l|c|c|c|}
\multicolumn{1}{c|}{ Estación } & Latitud & Longitud & $\begin{array}{c}\text { Gravedad } \\
\text { Absoluta }\end{array}$ \\
\hline CATACAMAS AA & $14^{\circ} 95^{\prime} 56.8^{\prime \prime} \mathrm{N}$ & $85^{\circ} 51^{\prime} 47.5^{\prime \prime} \mathrm{W}$ & $978266.336 \pm 0.005$ \\
\hline CATACAMAS D 26 & $14^{\circ} 49^{\prime} 48.531^{\prime \prime} \mathrm{N}$ & $85^{\circ} 51^{\prime} 47.420^{\prime \prime} \mathrm{W}$ & $978265.674 \pm 0.006$ \\
\hline SAN LORENZO AA & $13^{\circ} 25^{\prime} 06.5^{\prime \prime} \mathrm{N}$ & $87^{\circ} 26^{\prime} 55.5^{\prime \prime} \mathrm{W}$ & $978330.440 \pm 0.003$ \\
\hline SAN LORENZO CA & $13^{\circ} 25^{\prime} 06.6^{\prime \prime} \mathrm{N}$ & $87^{\circ} 26^{\prime} 55.0^{\prime \prime} \mathrm{W}$ & $978330.419 \pm 0.004$ \\
\hline SAN PEDRO SULAAA & $15^{\circ} 31^{\prime} 50.150^{\prime \prime} \mathrm{N}$ & $88^{\circ} 02^{\prime} 11.142^{\prime \prime} \mathrm{W}$ & $978387.134 \pm 0.005$ \\
\hline SAN PEDRO SULA BM & $15^{\circ} 31^{\prime} 49.597^{\prime \prime} \mathrm{N}$ & $88^{\circ} 02^{\prime} 11.243^{\prime \prime} \mathrm{W}$ & $978387.152 \pm 0.006$ \\
\hline SANTA ROSA DE COPÁN & $14^{\circ} 46^{\prime} 33.710^{\prime \prime} \mathrm{N}$ & $88^{\circ} 46^{\prime} 28.320^{\prime \prime} \mathrm{W}$ & $978081.850 \pm 0.004$ \\
AA & & & \\
\hline SANTA ROSA DE COPÁN & $14^{\circ} 46^{\prime} 33.710^{\prime \prime} \mathrm{N}$ & $88^{\circ} 46^{\prime} 28.320^{\prime \prime} \mathrm{W}$ & $978081.920 \pm 0.004$ \\
\hline TEGUCIGALPAAA & $14^{\circ} 05^{\prime} 14.660^{\prime \prime} \mathrm{N}$ & $87^{\circ} 09^{\prime} 34.069^{\prime \prime} \mathrm{W}$ & $978062.991 \pm 0.003$ \\
\hline TEGUCIGALPAAA & $14^{\circ} 05^{\prime} 13.402^{\prime \prime} \mathrm{N}$ & $87^{\circ} 09^{\prime} 33.203^{\prime \prime} \mathrm{W}$ & $978063.469 \pm 0.004$ \\
\hline
\end{tabular}

\section{MÉTODO}

La comparación entre el valor medido de la gravedad $g$ en un punto contra su valor teórico, suponiendo la Tierra como un cuerpo elipsoidal con densidad homogénea, resulta en diferencias o anomalías gravimétricas que pueden relacionarse con los valores locales de la densidad de las rocas. Estas mediciones de la gravedad local pueden interpretarse como la suma de varios efectos globales y regionales que generan contribuciones al valor de $g$ superiores en varios órdenes de magnitud a la gravedad producida por la estructura geológica local, la cual es el objeto de este estudio. Los cambios en las densidades dentro de los $5 \mathrm{~km}$ superiores de la corteza producirán variaciones de $g$ que no excederán el $0.01 \%$ de su valor en cualquier lugar, mientras que las fluctuaciones de la gravedad que puedan estar asociadas a cuerpos minerales con valor comercial probablemente no superarán $10^{-5} \mathrm{~g}$ (Burger et al., 2006). Estas estructuras geológicas contribuyen muy poco al valor total de $g$ pero esos efectos pueden ser detectados en el terreno utilizando gravímetros con 
sensibilidad de lectura de 0.01 mgals. Para poder separar estas contribuciones de la señal gravitacional global es necesario aplicar a las mediciones de $g$ una serie de correcciones geodésicas que eliminan los efectos de la elipticidad de la Tierra, rotación, altitud, mareas lunares y topografía. A partir de esta gravedad reducida, es posible formular hipótesis acerca del tipo de estructuras que mejor se ajustan a dichas observaciones utilizando inversión o modelos matemáticos directos.

\subsection{Corrección por efecto de latitud}

La rotación de la Tierra produce una aceleración centrífuga que actúa en dirección opuesta a la gravedad, y cuyos efectos dependen de la latitud. Otra dependencia de la latitud geográfica existe porque la Tierra no tiene simetría esférica, sino que se aproxima a un elipsoide de rotación. El modelo actual para el cálculo teórico de la gravedad de la Tierra sobre un punto en la superficie de un elipsoide de revolución, fue adoptado por la Asociación Internacional de Geodesia en 1967, y define la gravedad sobre un elipsoide de referencia que coincide aproximadamente con el nivel del mar, de acuerdo a la fórmula del sistema de referencia geodésico de 19670 GRS67 (Burger, et al.,2006, Heiskanen y Moritz, 1990):

$$
g_{n}=978.03185\left(1+0.005278895 \sin ^{2} \phi+0.000023462 \sin ^{4} \phi\right) c m \cdot s^{-2}
$$

Esta fórmula corrige por efectos de posición (latitud Ö) y aceleración centrífuga, y nos da el llamado valor de la gravedad normal, $g_{n}$.

\subsection{Corrección por efecto de elevación.}

La gravedad disminuye al incrementar la distancia al centro de la Tierra por efecto de la altura $z$ sobre el elipsoide de referencia. El gradiente de la gravedad $g$ con respecto a la distancia $R$ desde el centro del elipsoide, está dado por el siguiente modelo que recoge los efectos de la elipticidad del planeta, la rotación y la altura $z$ (Burger etal., 2006):

$$
d g / d R=-0.3086-0.00023 \cos 2 \phi+0.00000002 z
$$

\subsection{Corrección de Bouguer}

Esta corrección da cuenta de los efectos de la masa de material existente entre el punto de observación y el nivel del mar, asumiendo una placa infinita homogénea de un grosor igual a la elevación $z$ sobre el nivel del mar y una densidad de $\tilde{n}=2.67$ $\mathrm{g} / \mathrm{cm}^{2}$, que equivale a la densidad promedio de las rocas de la corteza terrestre 
(Burger etal., 2006, Heiskanen y Moritz, 1990). El valor de esta corrección es de:

$$
\Delta g_{B}=0.04193 \rho \mathrm{zGal} / \mathrm{m}
$$

el cual debe sustraerse del valor de la gravedad medido en el punto a una altura $z$ que se toma como la altura ortométrica con referencia al geoide.

\subsection{Corrección por efectos de topografía.}

Estas correcciones requieren un conocimiento local y regional del relieve del terreno que rodea la estación con el objetivo de obtener una corrección de Bouguer completa.

Las ondulaciones de la topografía con respecto al nivel de un punto de observación producen variaciones en el valor medido de $g$ debido a excesos y deficiencias de masa en la vecindad del punto de interés. Esta corrección se verifica por el método de Hammer (1939) que calcula los efectos gravitacionales que un anillo de grosor igual a la altura topográfica relativa $z$, densidad de la corteza $\tilde{n}$, radio interior $R_{i}$ y radio exterior $R_{0}$ produce en su centro, en donde se localiza la estación.

La ecuación que expresa la atracción gravitacional que produce el anillo en un punto situado en su centro y a nivel del fondo o a la cima del mismo es (Heiskanen y Moritz, 1990):

$$
g_{\text {anillo }}=2 \pi G \rho\left[R_{0}-R_{i}+\left(R_{i}^{2}+z^{2}\right)^{1 / 2}-\left(R_{0}^{2}+z^{2}\right)^{1 / 2}\right]
$$

Estos anillos se dividen en $n$ sectores uniformes, con atracción $g_{\text {ring }} / n$ debida a cada sector, y se calcula la gravedad debida a cada uno de ellos alrededor de la estación.

\subsection{Corrección por efectos de las mareas.}

Las mareas lunares producen variaciones en el valor de la gravedad, medido en un punto que resultan en cambios de $\pm 0.15 \mathrm{mGal}$ a partir de un valor promedio, y además pueden variar en el tiempo a razones de hasta $0.05 \mathrm{mGal} / \mathrm{hora}$.

Estos efectos pueden predecirse con exactitud y algunas veces ya aparecen incorporados al resultado de la medición según el tipo de instrumento utilizado, 0 bien puede hacerse uso de software especializado para calcular estos efectos (Rudman, Ziegler y Blakely, 1977). 


\subsection{Corrección por Deriva Instrumental}

Debido a factores inherentes al diseño del instrumento y a factores externos, como cambios de temperatura, presión, transporte, vibración, etc; cuando se realizan mediciones continuas de la gravedad con los gravímetros de muelle, como el utilizado en esta investigación, se observan diferentes valores para medidas realizadas en una misma localización pero en diferente tiempo. Esta desviación se conoce como la deriva instrumental. La deriva instrumental puede ser observada en cambios de periodo largo (meses), dependientes en gran medida de la edad y el uso del instrumento; o también en cambios de periodo corto (horas), dependientes estas últimas de los factores mencionados con anterioridad. Si el tiempo de observación es de pocas horas, se puede considerar que los cambios de periodo corto obedecen a un comportamiento lineal, por lo que el método de interpolación lineal resulta una buena aproximación a fin de realizar la corrección por deriva instrumental. (Cantos, 1974)

\section{OBTENCIÓN DE DATOS Y PROCESAMIENTO}

\subsection{Instrumentación}

La detección de las diferencias en el valor de la aceleración de la gravedad de un punto a otro requiere de la medición de cambios en el valor de $g$ de al menos 0.1 mGal. Para la determinación de la gravedad relativa, en esta investigación empleamos un gravímetro GRAVITON EG de la casa LaCoste \& Romberg. En dicho instrumento las pequeñas variaciones en la gravedad mueven una masa en contra de la fuerza restauradora de un resorte metálico de tipo longitud cero (Telford, W. M. et al, 1976), al cual se le han añadido transductores y convertidores A/D para lograr una resolución en los datos de 24 bit, equivalente a un tamaño por bit inferior a 0.001 $\mathrm{mGal}$ (LaCoste \& Romberg, 2002). El instrumento dispone de un mecanismo de nivelación automática, estabilidad térmica, y además proporciona corrección en tiempo real para las mareas gravitacionales. Las lecturas se hacen directamente a través de una pantalla VGA LCD o se descargan de la memoria FlashRAM de $32 \mathrm{MB}$ a través de los puertos seriales o USB (LaCoste \& Romberg, 2002). Los parámetros de medición del instrumento utilizados en esta campaña fueron: Tiempo de medida de 5 minutos, contador automático, error aceptado de $0.01 \mathrm{mGal}$, longitud de filtro de 30s, ningún retardo en lectura, ventana de error de 30s y nivel medio de ruido.

La posición es necesaria para la corrección por latitud de acuerdo a la ecuación (1). Asimismo, cambios en la elevación de la superficie terrestre conducen a variaciones en el valor de $g$ en función de la distancia al centro de la Tierra de 
acuerdo a la ecuación (2). Para poder corregir este efecto es preciso conocer la altura de cada punto con un error de al menos $25 \mathrm{~cm}$. (Burger et al, 2006). Para este propósito utilizamos un receptor de GPS de la casa Trimble, Modelo 5700, el cual es capaz de registrar las frecuencias L1 y L2 de la constelación de satélites de GPS (Blewitt, 1997) para rendir datos de posición con una precisión de 1 cm (Ver Figura 1).

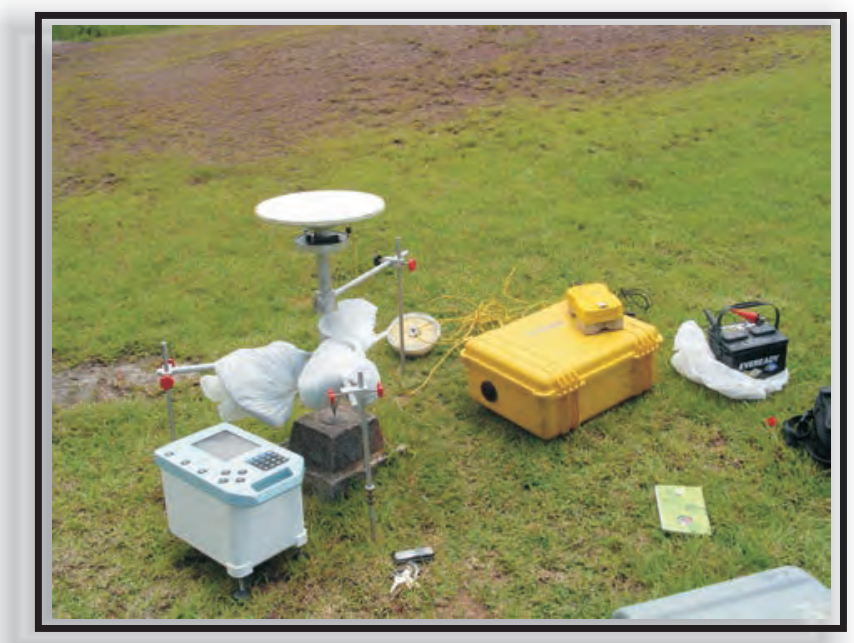

Figura 1. Gravímetro LaCoste \& Romberg GRAVITON EG en la estación NOAA-NGS de la UNAH. (caja con panel azul). Antena y receptor de GPS Trimble 5700. La batería de 12V alimenta al receptor de GPS.

\subsection{Obtención y procesamiento de datos de gravimetría}

Como resultado del trabajo de campo disponemos de datos de gravedad absoluta de alta precisión (0.01 miliGals). El gravímetro mide las diferencias entre la gravedad de cada estación y un valor interno de calibración o referencia, ó Äg . Conociendo asimismo esta lectura Äg para una estación base de referencia, en donde se conoce el valor absoluto de $g$ con gran exactitud, podemos determinar el valor absoluto de la gravedad en nuestra estación del campo utilizando la fórmula:

$$
g_{E O}=g_{E B}-\left(\Delta g_{E B}-\Delta g_{E O}\right)
$$

$g_{\mathrm{EO}} \quad$ gravedad absoluta en la estación de observación

$g_{E B} \quad$ gravedad absoluta en la estación base

$\Delta g_{E B:}$ lectura del gravímetro en la estación base

$\Delta g_{\mathrm{EO}}$ : lectura del gravímetro en la estación de observación 
En nuestro caso, utilizamos la estación NOAA-NGS de Tegucigalpa localizada en los predios del Observatorio Astronómico de la UNAH, donde $g=978063.469 \pm$ 0.004 mGals (N.O.A.A, 2001).

En la campaña que realizamos en esta investigación se operó en circuitos, siguiendo las técnicas expuestas en Seigel (1995), a fin de poder aplicar interpolación lineal y corregir para la deriva instrumental que ocurre a lo largo del circuito. Se comienza de una estación base y se regresa a ella en intervalos de tiempo de dos a tres horas, formando un polígono cerrado, como se ilustra para un circuito típico en la Figura 2. La metodología de recolección de datos seleccionada en esta investigación es la que comúnmente se utiliza en este tipo de estudios, en el cual se cuenta con un solo gravímetro y condiciones restringidas de tiempos de medición. (Burger et al, 2006).

CAMPAÑA 5 JULIO 2007. ESTACION BASE SUYAPA -UNAH

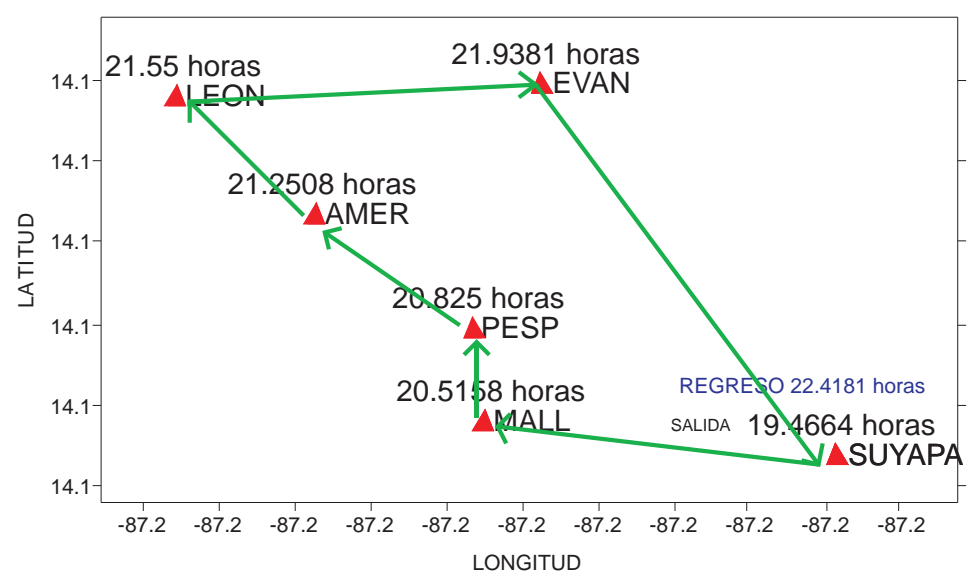

Figura 2. Esquema que ilustra la forma es que se recolectaron los datos para cada circuito. El punto inicial y final de circuito fue la estación base de gravedad absoluta conocida. El tiempo transcurrido para cada circuito fue de dos a tres horas, a fin de suponer un incremento lineal de la deriva instrumental

\subsubsection{Red Gravimétrica y enlace de estaciones.}

Por la extensión geográfica de la zona de influencia y por la dificultad de acceso a cada una de las estaciones de observación seleccionadas, a fin de realizar medidas en intervalos de tiempo aceptables en la estación base, en vez de contar con una sola estación base, se estableció una red de 10 estaciones base, interconectadas a 
través de una base principal (Figura 3). La base principal fue la estación Suyapa de la UNAH, que fue escogida como punto fijo, ya que ésta forma parte de una red regional a nivel de Centro América en donde se ha determinado la aceleración absoluta de la gravedad (Nacional Ocean Service and National Geodetic Survey, 2001).

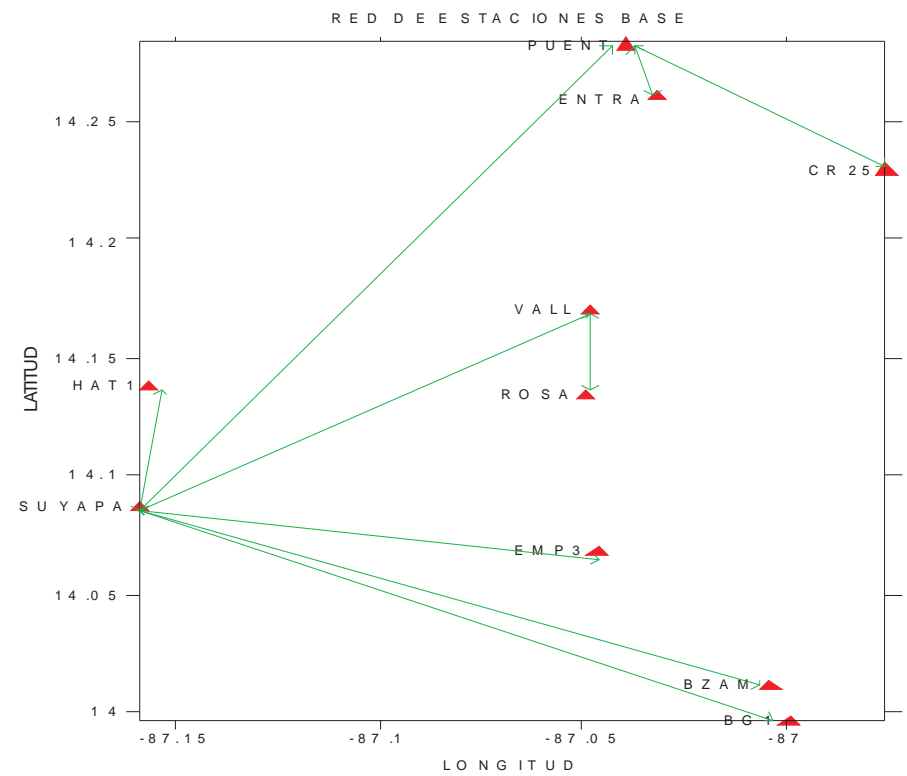

Figura 3. Mapa que indica la localización de las estaciones base, mediante esta red de estaciones base todas las estaciones de observación están ligadas al punto fijo de la estación NOAA-NGS de Tegucigalpa en el Observatorio a de Suyapa en la UNAH (NOAA, 2001), en donde el valor de la gravedad absoluta es conocido.

\subsubsection{Determinación y corrección de deriva instrumental}

Con el propósito de corregir la deriva instrumental, realizamos mediciones de la gravedad en diferentes tiempos en la estación base de cada circuito, seleccionamos como valor de referencia la primera medida realizada en el circuito. Asumiendo un comportamiento lineal de la gravedad con el tiempo $\mathrm{g}(\mathrm{t})$, mediante interpolación lineal, obtuvimos el valor de la gravedad en la estación base para los tiempos en los que se midió la gravedad en las otras estaciones de observación, de acuerdo a la siguiente ecuación: 


$$
g_{t}=g_{0}+\left(g_{f}-g_{0}\right)\left(\frac{t-t_{0}}{t_{f}-t_{0}}\right)
$$

Donde: $\boldsymbol{g}_{\mathbf{t}}$ : valor de gravedad en la estación base interpolado; $\boldsymbol{t}$ : tiempo en el que se midió la gravedad en la estación de observación; $\boldsymbol{g}_{0}$ : valor de gravedad en la estación base al inicio del circuito; $\boldsymbol{t}_{0}$ : tiempo de inicio del circuito; $\boldsymbol{g}_{\boldsymbol{f}^{*}}$ valor de gravedad en la estación base al cerrar el circuito; $\boldsymbol{t}_{\mathrm{f}}$ : tiempo de cierre del circuito.

La diferencia observada entre el valor interpolado y el valor de referencia proporcionó la deriva instrumental, que se sumó o restó al valor obtenido en cada una de las estaciones de observación, según fuese el caso: Cuando se obtuvieron diferencias positivas, estas se restaron y cuando se obtuvieron diferencias negativas, éstas fueron sumadas al valor de la estación correspondiente.

En las figuras 4 y 5 se grafican los resultados obtenidos al aplicar el método con los valores del circuito de la figura 2. En la Tabla 2 se observan los resultados obtenidos al aplicar la interpolación lineal para hacer las correcciones por deriva del instrumento. El tiempo máximo invertido en el recorrido de los circuitos en este estudio fue de 3.9942 horas y el mínimo fue de 0.7997 horas. En las tablas correspondientes a las áreas de estudio se dan a conocer los resultados obtenidos por deriva instrumental.

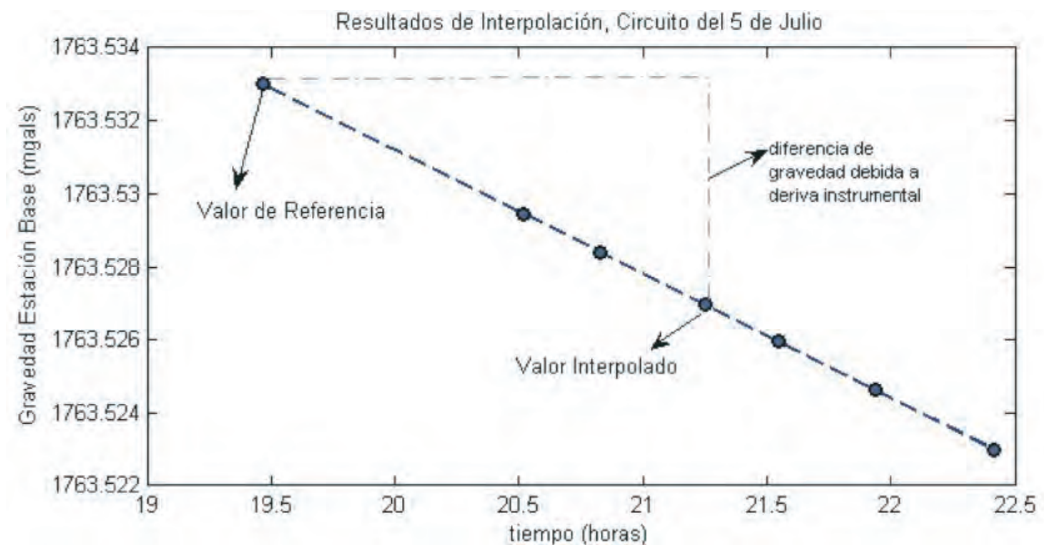

Figura 4. Resultados de interpolación lineal para valores de la estación base se Suyapa. Los valores inicial y final en el eje "y" corresponden a las mediciones realizadas al inicio y final del circuito respectivamente, los valores restantes son las interpolaciones correspondientes a los tiempos en que se realizó la medición en cada una de las estaciones de observación. 

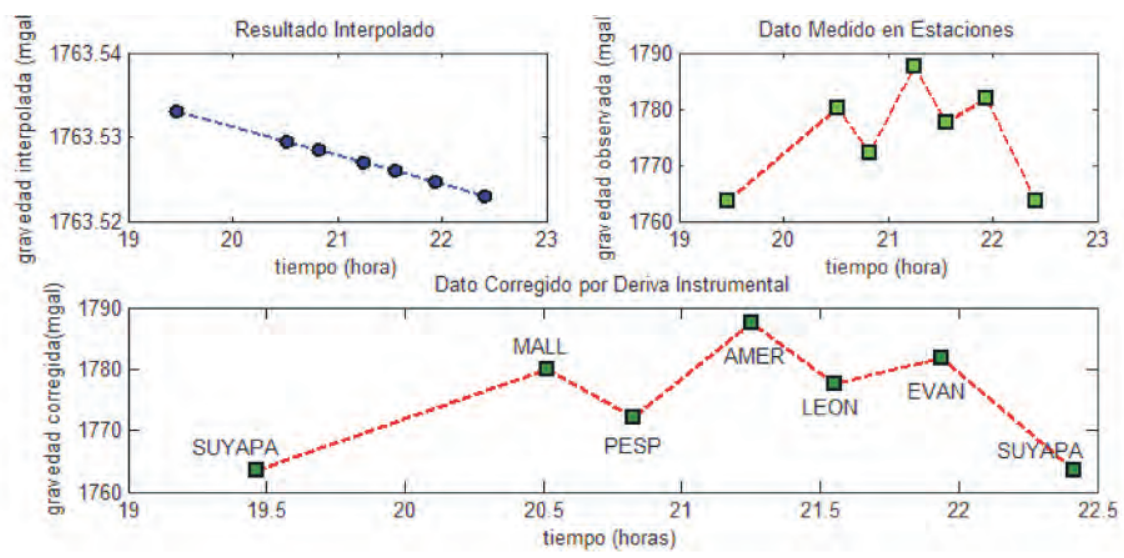

Figura 5. Los resultados de la interpolación para la estación base se indican en azul, no se observa mucha diferencia en ellos por la escala utilizada en el gráfico. La corrección por deriva en este circuito fue muy pequeña a tal grado que el valor real y corregido se grafican en el mismo punto.

\begin{tabular}{|c|c|c|c|c|}
\hline ID & $\begin{array}{c}\text { Lec. } \\
\text { Grav. } \\
\text { mgals }\end{array}$ & $\begin{array}{c}\text { Tiempo } \\
\text { (hora GMT) }\end{array}$ & $\begin{array}{c}\text { Deriva } \\
\text { Inst. } \\
\text { mgals }\end{array}$ & $\begin{array}{c}\text { Valor } \\
\text { corregido }\end{array}$ \\
\hline SUYAPA & 1763.533 & 19.4664 & & 1763.533 \\
\hline MALL & 1780.080 & 20.5158 & -0.001 & 1780.081 \\
\hline PESP & 1772.199 & 20.8250 & -0.001 & 1772.200 \\
\hline AMER & 1787.485 & 21.2508 & -0.001 & 1787.486 \\
\hline LEON & 1777.649 & 21.5500 & -0.001 & 1777.650 \\
\hline EVAN & 1781.763 & 21.9381 & -0.008 & 1781.771 \\
\hline SUYAPA & 1763.523 & 22.4181 & -0.010 & 1763.533 \\
\hline
\end{tabular}

Tabla 2. Circuito formado con estación base de Suyapa y 5 estaciones de observación. Los valores negativos de deriva indican que el valor de referencia fue

superior al valor final en la estación base, por lo que la corrección se realizó sumando la deriva al valor de observación. El tiempo total del circuito fue de 2.9517 horas con una deriva máxima de $-0.010 \mathrm{mgal}$.

\subsubsection{Corrección por efecto de mareas, latitud, elevación y Bouguer.}

Las correcciones para compensar los cambios de la gravedad debidos a las mareas lunares, son proporcionadas automáticamente por el instrumento empleado (GRAVITON EG). Las correcciones por efectos de latitud, altura, corteza de Bouguer se calculan directamente de las ecuaciones (1), (2), y (4). 


\subsubsection{Corrección Topográfica.}

Utilizamos el programa HAMXYZ2 ${ }^{\mathrm{TM}}$ de Gradient Geophysics que permite hacer correcciones de topografía con ayuda de Modelos de Elevación Digital (DEMs) en formato ASCII, según el método de Hammer (1939) y la ecuación (5). Existen archivos digitales de este tipo a nivel global proporcionados por el Servicio Geológico de los Estados Unidos (USGS) que pueden obtenerse en el sitio http://seamless.usgs.govl. Estos modelos son el resultado de las misiones de topografía de los transbordadores espaciales de NASA (Shuttle Radar Topography Mission).

Para correcciones en estudios como el nuestro, deben tomarse en cuenta los accidentes topográficos del terreno a varios metros de cada estación así como la topografía (montañas, grabens, océanos, etc.) dentro de un radio de varios kilómetros. Los efectos mayores son los primeros, corresponden a los sectores cercanos al punto de interés. Leaman (1988) reporta errores en exceso de $0.1 \mathrm{mgals}$ debidos a ondulaciones del terreno en la vecindad de las estaciones. Dichos efectos han sido estimados tradicionalmente mediante apreciaciones visuales en el campo y son por su naturaleza, inexactos y cualitativos, ya que dependen de la experiencia del observador (Schiavone et al., 2007). Los modelos de elevación digital disponibles para Honduras tienen apenas una resolución de $90 \mathrm{~m}$ horizontales y $10 \mathrm{~m}$. verticales; por tanto, utilizamos correcciones de topografía solamente para distancias desde $390 \mathrm{~m}$ hasta aproximadamente $22 \mathrm{~km}$ de cada estación. Los parámetros de los anillos de Hammer que utilizamos en este estudio se resumen en la Tabla 4:

\begin{tabular}{|l|l|l|l|}
\hline Zona & $\begin{array}{c}\text { Radio } \\
\text { interior } \\
(\mathrm{m})\end{array}$ & $\begin{array}{c}\text { Radio } \\
\text { exterior } \\
(\mathrm{m})\end{array}$ & $\begin{array}{c}\text { No. } \\
\text { Secto } \\
\text { res }\end{array}$ \\
\hline F & 390 & 895 & 8 \\
\hline G & 895 & 1529 & 12 \\
\hline H & 1529 & 2614 & 12 \\
\hline I & 2614 & 4469 & 12 \\
\hline J & 4469 & 6652 & 16 \\
\hline K & 6652 & 9902 & 16 \\
\hline L & 9902 & 14741 & 16 \\
\hline M & 14741 & 21943 & 16 \\
\hline
\end{tabular}

Tabla 4. Anillos de Hammer para HAMXYZ2 ${ }^{\mathrm{TM}}$ 
Para investigar la magnitud de los efectos de la densidad del terreno sobre las correcciones, hicimos cálculos con densidades de 2.67, 2.60, y $2.55 \mathrm{~g} / \mathrm{cm}^{3}$ En la figura 5 se muestran los efectos de la topografía para dos valores extremos de la densidad de la corteza para la región del Zamorano. Como es de esperar, las estaciones ubicadas en el centro del valle exhiben efectos menores, entre 1.5 y 2.5 mgals, comparadas con las estaciones en las zonas montañosas que sufren efectos fuertes de hasta aproximadamente 4mgals. Las Figuras 8, 11, 16 y 19 reproducen estos cálculos para las regiones de estudio de Tegucigalpa, Valle del Zamorano, Valle de Cantarranas y Valle de Morocelí, respectivamente. Efectos de topografía relativamente bajos, menores de 1.0 mgals, se consideran ideales para resolver anomalías de Bouguer locales. En nuestro caso existen correcciones fuertes, superiores a los 10 mgals, en regiones montañosas, y correcciones relativamente altas, entre 1.0 y 3.0 mgals, en los valles y depresiones topográficas de las zonas de estudio.

Debido a que las correcciones de topografía se suman a la anomalía de bouguer, y a que el programa HAMXYZ2 asume un valor uniforme de la densidad de la corteza de $2.67 \mathrm{~g} . \mathrm{cm}^{-2}$ en toda el área alrededor de la estación, se producirán artefactos en las correcciones de bouguer finales en áreas de topografía montañosa y en las zonas próximas a ellas, las cuales tendrán valores de bouguer sobreestimados en varios mgals. Considerando las limitaciones de nuestros modelos de elevación digital y las restricciones de la Tabla 4 consideramos los valores de la gravedad residual en esos puntos con cierta reserva, limitando el análisis para regiones donde el valor de las correcciones de topografía sea uniforme e inferiores a 2 mgals.

Mapa Topográfico del Valle del Zamorano

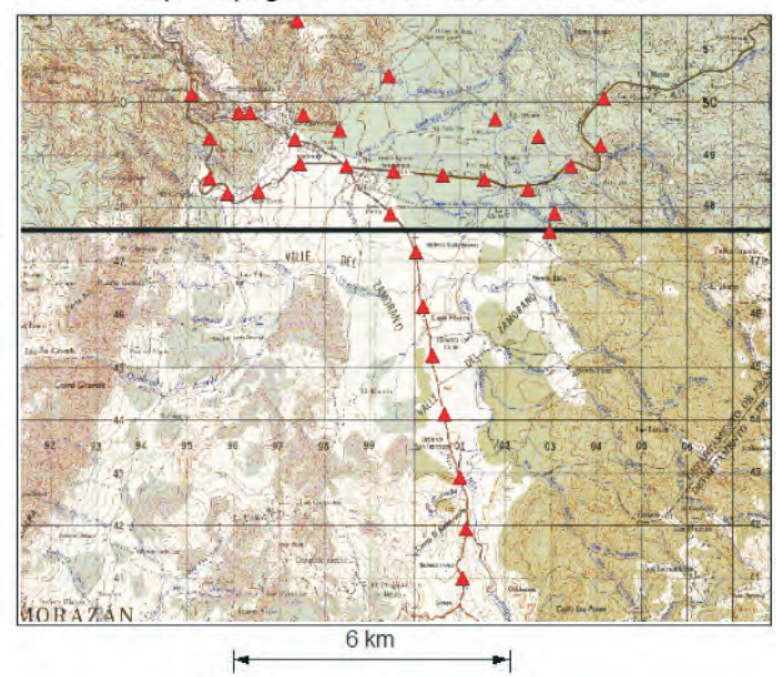

Dirección de Investigación Científica $\longdiv { 4 1 }$ 


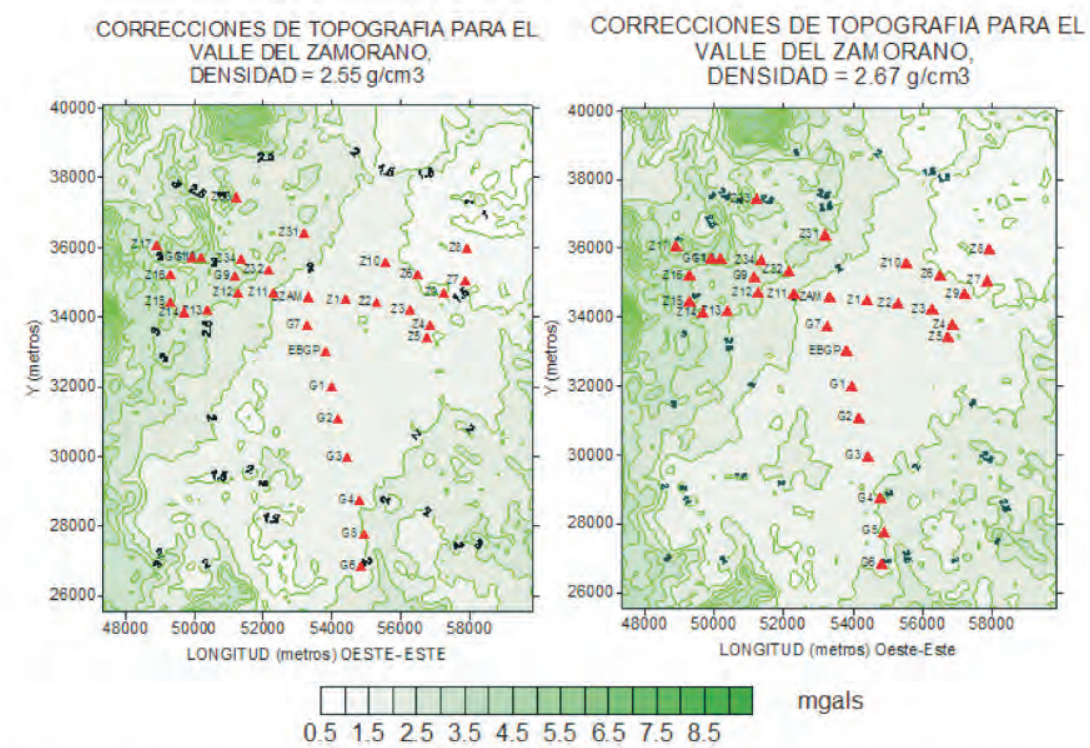

Figura 6. Efectos gravitacionales de la topografía para el Valle del Zamorano asumiendo densidades de corteza de $2.55 \mathrm{~g} / \mathrm{cm}^{3}$ y $2.67 \mathrm{~g} / \mathrm{cm}^{3}$. Para dibujar estos contornos se construyó una malla de 10,000 puntos para esta región a partir de los modelos de elevación digital (SRTM), y para cada punto se calcularon correcciones con el programa HAMXYZ2 y el método de Hammer (1939), utilizado las zonas descritas en

la Tabla 4. Los triángulos rojos representan la ubicación de las estaciones.

\section{Cálculo de la anomalía de Bouguer}

Finalmente, compensamos a los valores de gravedad absoluta calculados mediante (5) las correcciones gravimétricas obtenidas de las ecuaciones (1), (2), (3), y (4). Obtuvimos valores reducidos de g, o anomalías de Bouguer, los cuales pueden resultar positivos (excesos locales de gravedad) o negativos (deficiencias locales de gravedad), según la contribución relativa de cada uno de los efectos arriba mencionados:

$$
\Delta g_{B}=g_{o b s}-g_{n}+F A_{c o r r}-B_{c o r r}+T C
$$

Donde: $g_{B}$ : Anomalía de Bouguer; $g_{\text {obs: }}$ gravedad absoluta observada; $g_{n}$ : gravedad normal (Ecuación 1); $F A_{\text {corr }}$ : Corrección de aire libre (Ecuación 2); $B_{\text {corr: }}$ Corrección de Bouguer (Ecuación 3); TC: Corrección de topografía (Burger, et. al., 2006). Las deficiencias en el valor de la gravedad (baja densidad) pueden corresponder a domos de sal, cavernas, o presencia de hidrocarburos, mientras que un exceso de gravedad (alta densidad) puede deberse a un cuerpo altamente mineralizado, entre 
otras posibilidades. Estas anomalías de Bouguer, junto al conocimiento adicional de la geología local, pueden ser utilizadas para investigaciones en exploración geotécnica, minera, petrolera, aguas subterráneas, geometría de cuencas sedimentarias y estudios arqueológicos.

\subsection{Procesamiento de datos de posición utilizando el Sistema de Posicionamiento Global (GPS).}

Registramos las señales de la constelación de satélites del GPS en la tarjeta interna de un receptor Trimble 5700 (TrimbleTM, 2002) y luego utilizamos el puerto serial de éste para trasladarlas a una computadora y convertir el formato propietario de la casa Trimble al formato RINEX (Receiver Independent Exchange Format) con el objeto de procesar la información de la señal del GPS y obtener posición. La teoría del Sistema de Posicionamiento Global, el tipo de señales empleadas y las técnicas matemáticas requeridas para obtener coordenadas pueden revisarse en Blewitt, (1997). En la práctica se requiere de un pre-procesamiento de los datos para evaluar la calidad de la señal y finalmente un procesamiento para obtener posición. El software requerido es facilitado por el consorcio de instituciones de investigación geofísica UNAVCO (www.unavco.org). Para pre-procesamiento existe el programa TEQC (Estey, L.H. y C.M. Meertens ,1999) el cual hace traslación de formatos, edición y control de calidad. Para procesamiento existe el software OPUS (Online Positioning User Service) que permite obtener posición con errores del orden de 1 cm. (http://www.ngs.noaa.gov/OPUS). En particular nos interesa además de la Latitud y Longitud de cada estación, la altura elipsoidal proporcionada directamente por el GPS (ver Li y Gotze, 2001) para ser utilizadas en las ecuaciones (1) y (2) de las correcciones por posición y por altura, respectivamente.

\section{RESULTADOS}

Después de aplicar las correcciones gravimétricas a los datos de gravedad absoluta en cada región, obtuvimos fuertes anomalías negativas de Bouguer, entre -58 y -80 mgals, para todas las zonas de influencia de nuestro estudio, que son: Área metropolitana de la ciudad de Tegucigalpa, el Valle del Zamorano, y el Valle de San Juan de Flores (Cantarranas) y Morocelí. La magnitud de estas anomalías es consistente con los valores regionales reportados anteriormente por Bowin (1978) y Weyl (1980) en regiones montañosas de Honduras, indican una deficiencia de masa a nivel regional que puede interpretarse con modelos de compensación isostática. Dentro de la señal regional de la anomalía de Bouguer existen variaciones locales de menor longitud de onda debidas a la estructura geológica en los primeros $5 \mathrm{~km}$ de la corteza. Analizamos estas variaciones y obtuvimos información acerca de estructura geológica en zonas selectas. 


\subsection{AREA METROPOLITANA DE LA CIUDAD DE TEGUCIGALPA}

La Figura 7 muestra la localización de las estaciones dentro del área metropolitana de la ciudad de Tegucigalpa. Los valores, ajustes de los datos experimentales, las correcciones gravimétricas y anomalías de Bouguer se muestran en las Tablas 5 y 6 , respectivamente. El área metropolitana de Tegucigalpa sufre correcciones por efectos de topografía relativamente fuertes (Figura 8), de hasta 5 mgals en la zona cercana al Hatillo y Santa Lucía en el límite norte y oriental, mientras que hacia el centro y sur de la ciudad las correcciones son pequeñas y menos irregulares, oscilando entre 0.4 y 1.6 mgals. En la Figura 8 se observa que la mayoría de las estaciones del centro, sur y occidente de Tegucigalpa exhiben correcciones de topografía con valores muy cercanos entre si.

Esto significa que los artefactos inherentes al cálculo de las correcciones de topografía, debidos a la sobreestimación y homogeneización de la densidad del terreno, son relativamente uniformes en la zona y no alteran significativamente la forma de las curvas de gravedad residual. El mapa de contorno de las anomalías de Bouguer para Tegucigalpa se muestra en la Figura 9, mostrando un declive suave hacia el Occidente de la ciudad, consistente con una disminución en la densidad hacia esa dirección. Una comparación entre el contorno de las anomalías de Bouguer y la geología de Tegucigalpa se observa en la Figura 10, en la cual existe una alta correlación entre ambas. La cresta alrededor de -60 mgals que se observa en la Figura 9 y en el mapa de contorno de la Figura 10b es la señal debida a la formación Río Chiquito (Krc) del grupo de Valle de Ángeles. Este consiste de capas rojas de textura fina, vestigios de un abanico aluvial cretácico, contiene lutitas, limonitas, areniscas y conglomerados de cuarzo (Rogers, R.D. y E.A. O'Conner, 1993).

De acuerdo a estos autores, el espesor de estas capas varía aproximadamente entre 400 y 800 metros. Hacia el Occidente y Sur de Tegucigalpa dominan principalmente tres formaciones del grupo Padre Miguel: Miembro Cerro Grande (Tcg), consistente en secuencias gruesas de ignimbritas, tobas y sedimentos volcánicos (Williams y Mc Birney, 1969); Secuencia de ignimbritas (Tpm) con tobas riolíticas y andesíticas, y el Miembro El Periodista (Tep), que exhibe sedimentos volcanoclásticos de arenisca, grava y limonita (O'Conner, 1986) con espesor de unos $100 \mathrm{~m}$ aproximadamente (Rogers, R.D. y E.A. O'Conner, 1993). En el mapa de contorno de las anomalías de Bouguer (Figuras 9 y 10b) este grupo produce una depresión con valores bajos entre -70 y -80 mgals hacia el Sur y Occidente de la ciudad. 
Nuestra exploración preliminar del campo gravitacional en el área de Tegucigalpa, a pesar de la baja densidad de estaciones, muestra una correlación fuerte entre la geología del cuadrángulo y las anomalías de Bouguer. El contraste máximo entre la señal gravitacional de la formación Río Chiquito y la correspondiente al grupo Padre Miguel es de alrededor de 20 mgals, lo cual significa que es posible definir un nivel de gravedad regional asociada al contacto entre estas dos formaciones. Al observar en la Tabla 6 las anomalías de Bouguer para las estaciones localizadas en la frontera entre la formación Río Chiquito y los miembros del Grupo Padre Miguel hacia el Occidente y Sur de la ciudad ( CTRY, YIP, COHC, UPN, TRAP, HATO) es evidente que tienen todas valores muy cercanos a -60 mgals, los hechos anteriores están asociados a un nivel de gravedad regional adecuado para explorar el grosor de la formación Río Chiquito, un parámetro de gran importancia geotécnica para la industria local de la construcción.

Por otra parte, valores negativos inferiores a - 75 mgals hasta extremos cercanos a los -80 mgals son aparentes hacia el extremo occidental de la ciudad especialmente en las estaciones PPOR, LPED, COPE, VELE, CATO con formaciones de tobas y sedimentos volcánicos del Grupo Padre Miguel. La densidad de nuestras estaciones es insuficiente para calcular una superficie de gravedad regional como ajuste de mínimos cuadrados a una superficie de contornos de anomalías de Bouguer. El promedio de.72 las anomalías de Bouguer en Tegucigalpa a partir de la Tabla 6 es de -66mgals. Una inspección de la Figura 9 sugiere un valor de gravedad regional en la vecindad de -67 mgals, específico para la zona metropolitana de Tegucigalpa como punto de partida para futuros trabajos de exploración geofísica en lazona.

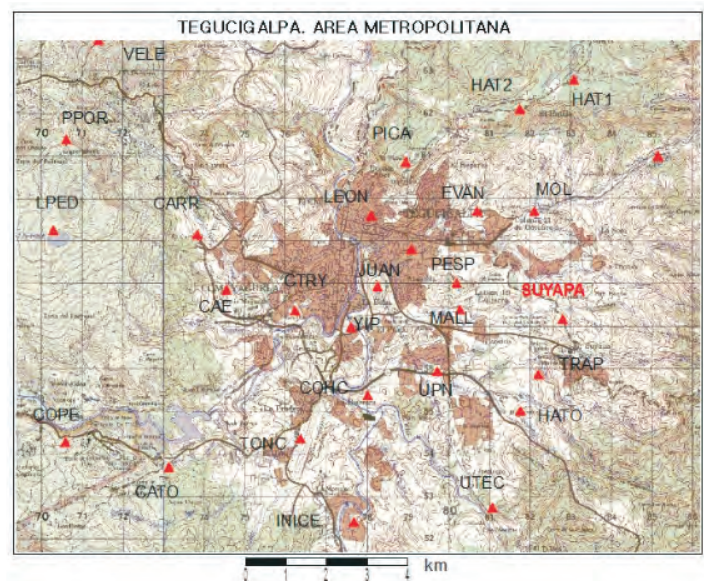

Figura 7. Localización de las estaciones de gravimetría (triángulos rojos) dentro del área metropolitana de la ciudad de Tegucigalpa, Honduras 
Tabla 5. Datos experimentales para Tegucigalpa

\begin{tabular}{|c|c|c|c|c|c|}
\hline Estación & 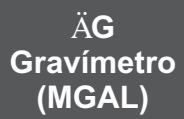 & Error & $\begin{array}{l}\text { RMS } \\
\text { Error }\end{array}$ & $\begin{array}{c}\text { Corrección } \\
\text { de Marea }\end{array}$ & $\begin{array}{c}\text { Deriva } \\
\text { Instrumental }\end{array}$ \\
\hline SUYAPA & 1764.080 & & 0.125 & -0.065 & \\
\hline UTEC & 1762.066 & & 0.057 & -0.131 & -0.332 \\
\hline $\mathrm{COHC}$ & 1776.529 & & 0.388 & -0.388 & -0.001 \\
\hline INICE & 1771.713 & & 0.025 & -0.092 & -0.002 \\
\hline TONC & 1773.048 & 0.005 & 0.031 & 0 & 0.002 \\
\hline CATO & 1754.733 & 0.013 & 0.161 & -.0 .021 & 0.004 \\
\hline COPE & 1737.165 & 0.006 & 0.038 & -0.052 & 0.006 \\
\hline MALL & 1780.080 & 0.002 & 0.038 & -0.116 & -0.001 \\
\hline PESP & 1772.199 & 0.006 & 0.08 & -0.122 & -0.001 \\
\hline AMER & 1787.485 & 0.008 & 0.024 & -0.125 & -0.001 \\
\hline LEON & 1777.649 & 0.007 & 0.027 & -0.125 & -0.001 \\
\hline EVAN & 1781.763 & 0.003 & 0.052 & -0.123 & -0.008 \\
\hline MOL & 1778.385 & 0.001 & 0.02 & -0.107 & -0.086 \\
\hline LSTA & 1757.416 & 0.017 & 0.045 & -0.098 & -0.151 \\
\hline ELEN & 1727.570 & 0.006 & 0.045 & -0.089 & -0.203 \\
\hline UPN & 1782.772 & 0.007 & 0.026 & 0.041 & -0.135 \\
\hline JUAN & 1763.785 & 0.003 & 0.021 & 0.036 & -0.135 \\
\hline YIP & 1791.548 & 0.007 & 0.06 & 0.028 & -0.322 \\
\hline HATO & 1760.840 & 0.004 & 0.031 & 0.018 & -0.438 \\
\hline TRAP & 1755.987 & 0.005 & 0.28 & 0.007 & -0.520 \\
\hline C1CG & 1757.306 & 0.003 & 0.028 & 0.04 & -0.117 \\
\hline CGZ8 & 1750.393 & 0.007 & 0.041 & 0.048 & -0.145 \\
\hline VELE & 1678.197 & 0.002 & 0.024 & 0.057 & -0.233 \\
\hline PPOR & 1663.928 & 0.005 & 0.035 & 0.062 & -0.300 \\
\hline CRTY & 1775.166 & 0.006 & 0.041 & -0.143 & -0.210 \\
\hline CARR & 1749.854 & 00.02 & 0.027 & -0.131 & 0.299 \\
\hline CAES & 1757.755 & 0.003 & 0.043 & -0.112 & 0.412 \\
\hline PICA & 1715.348 & 0.012 & 0.025 & -0.132 & 0.007 \\
\hline HAT1 & 1685.550 & 0.005 & 0.045 & -0.114 & 0.012 \\
\hline HAT2 & 1684.743 & 0.008 & 0.025 & -0.099 & 0.014 \\
\hline TABL & 1663.529 & 0.005 & 0.024 & -0.029 & -0.005 \\
\hline EMP & 1639.194 & 0.004 & 0.027 & -0.009 & -0.008 \\
\hline LUCI & 1679.892 & 0.003 & 0.083 & 0.016 & -0.013 \\
\hline LPED & 1659.366 & 0.003 & 0.042 & -0.026 & -0.337 \\
\hline OLI & 1767.889 & 0.005 & 0.02 & -0.004 & 0.003 \\
\hline
\end{tabular}


Tabla 6. Correcciones gravimétricas y Anomalía de Bouguer Tegucigalpa.
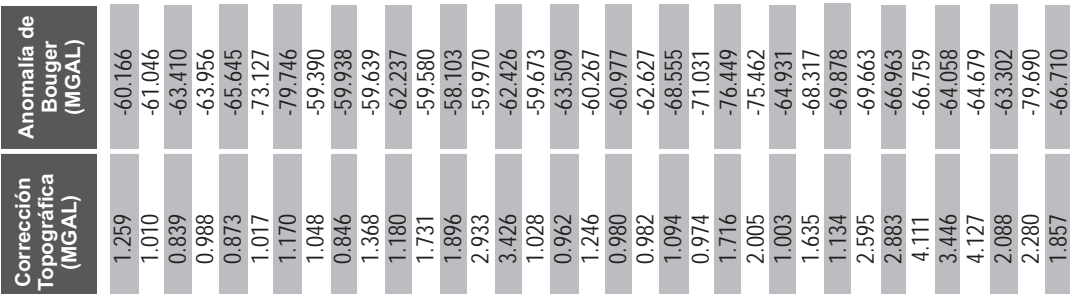

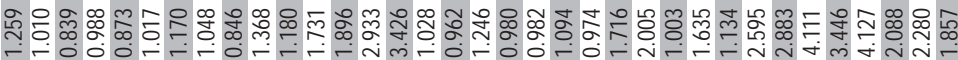

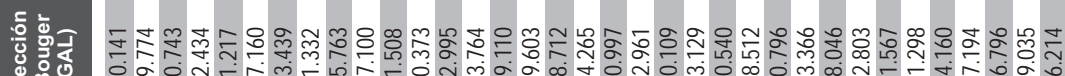

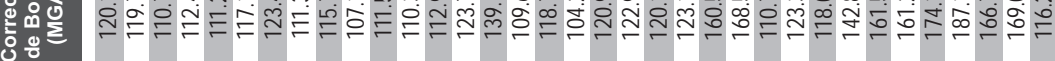

:응 혼

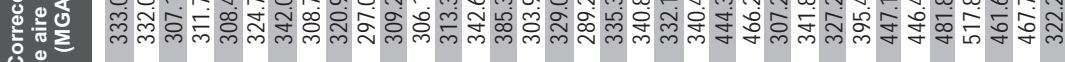

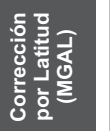

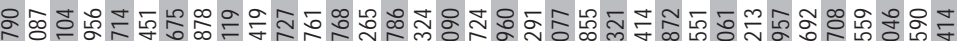
స్

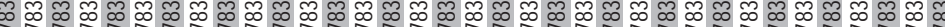

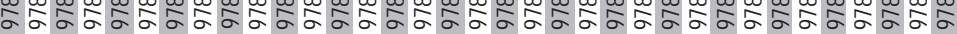

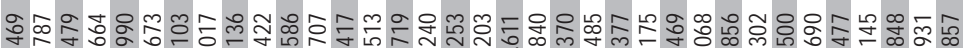

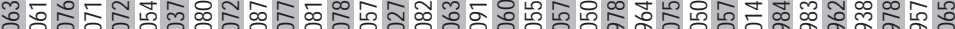

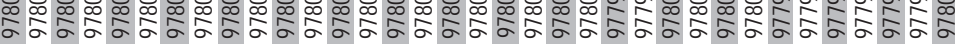

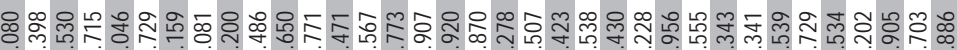

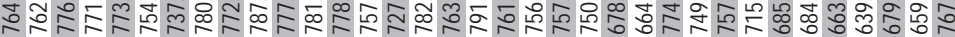




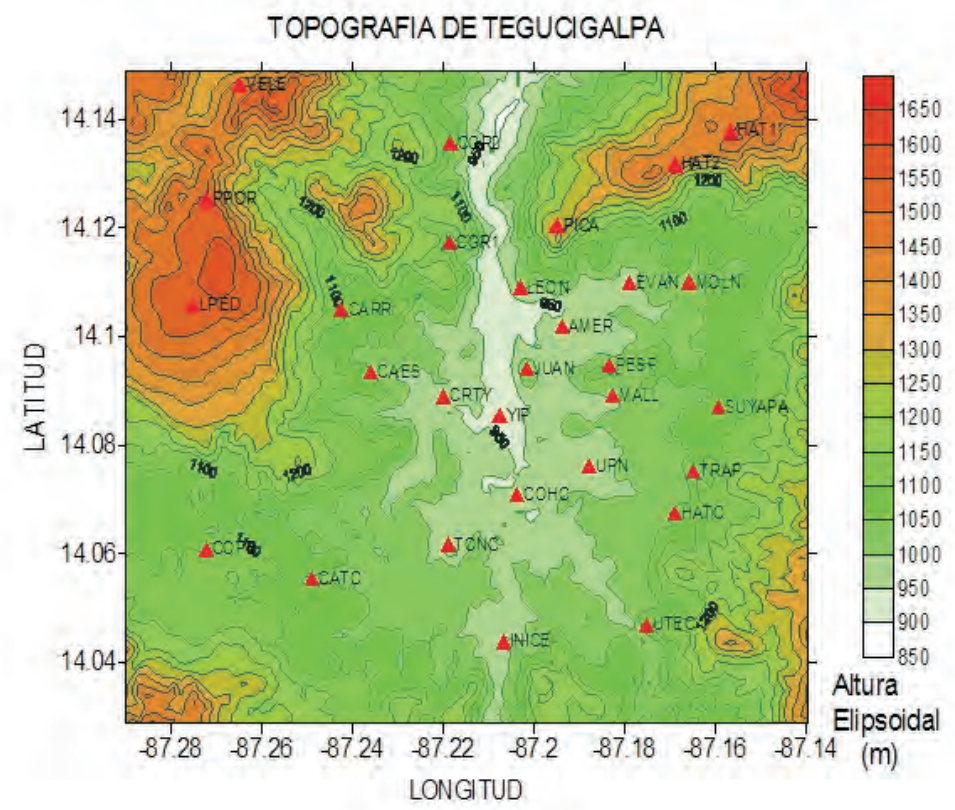

a

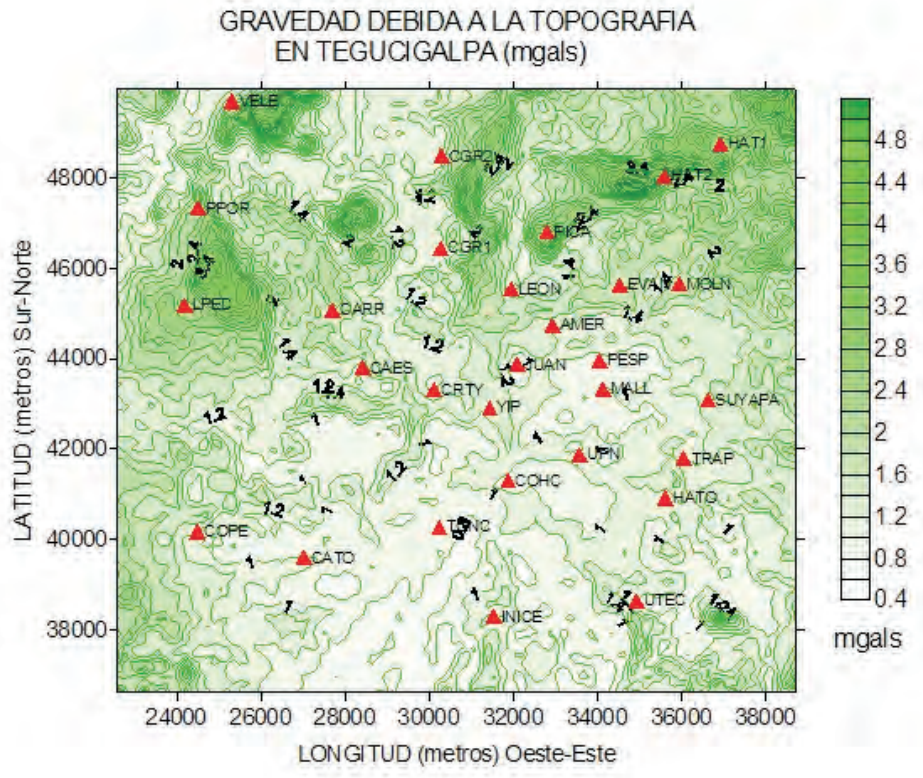

b

Figura 8. (a) Modelo de elevación digital SRTM y (b) Correcciones de topografía para la ciudad de Tegucigalpa. 


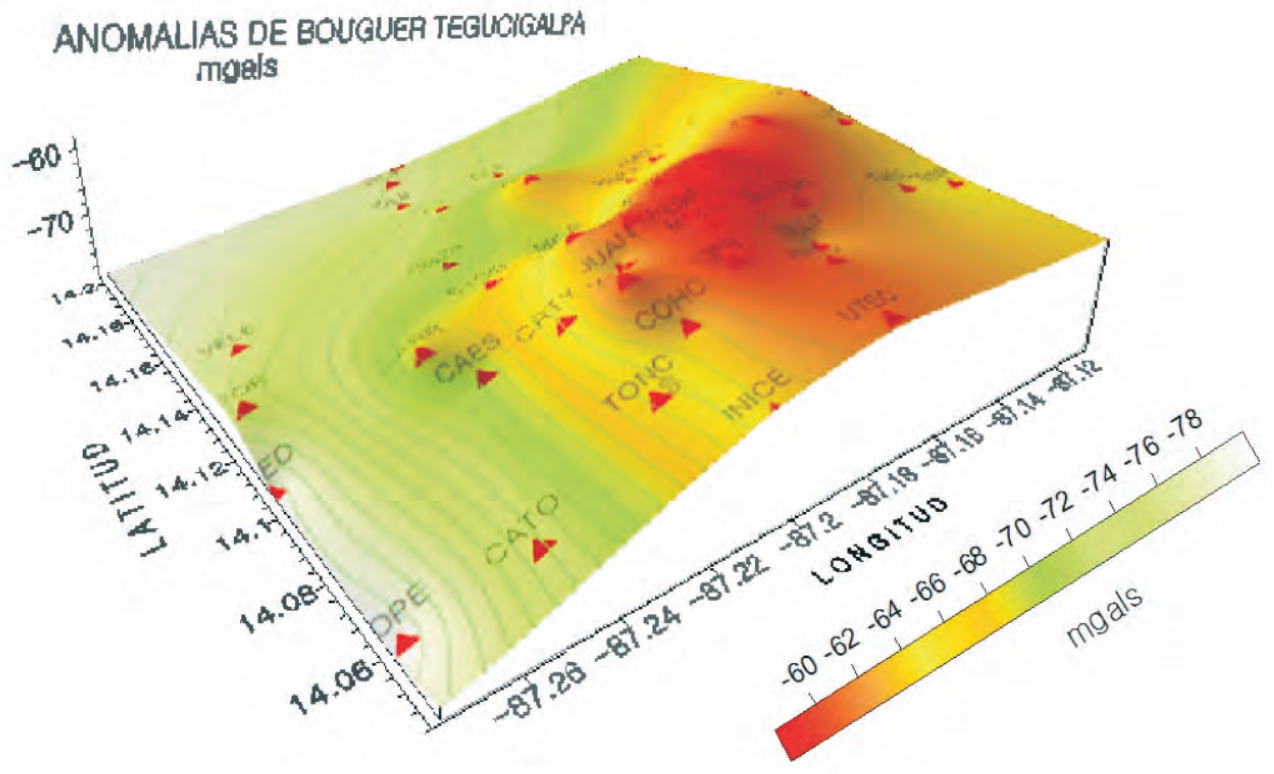

Figura 9. Mapa de contorno en 3D de las anomalías de Bouguer para el área metropolitana de la ciudad de Tegucigalpa. ADVERTENCIA: Ignorar los líneas de contorno en regiones donde no hay estaciones. 


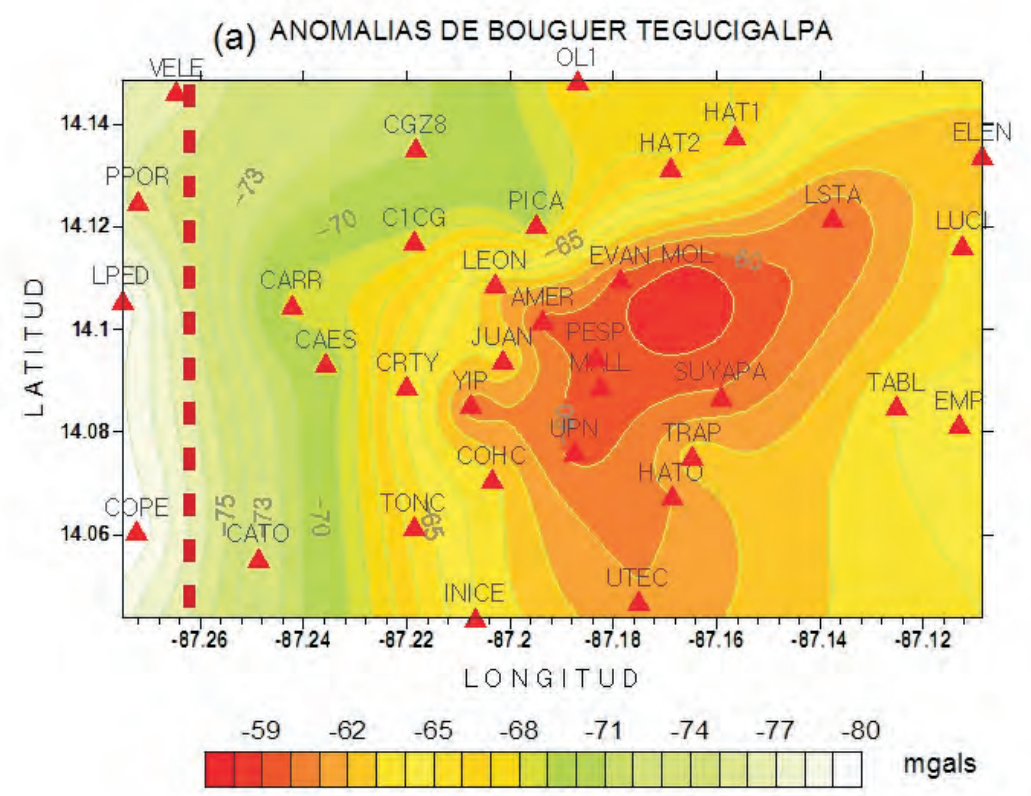

(b) GEOLOGIA DE TEGUCIGALPA

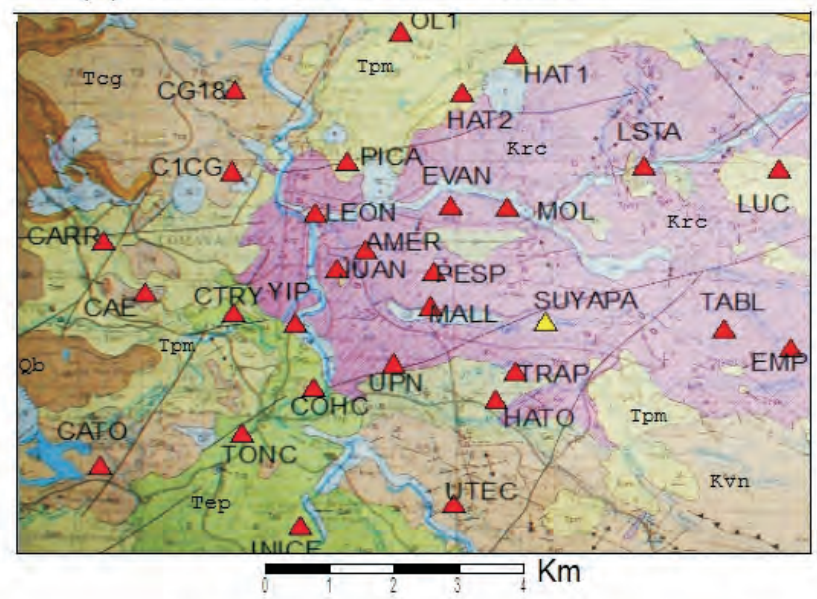

Figura 10. (a) Mapa de contornos de las anomalías de Bouguer para el área metropolitana de la Ciudad de Tegucigalpa, Honduras. ADVERTENCIA: Ignorar las líneas de contorno en las regiones donde no hay estaciones. (b) sección del Mapa geológico del cuadrángulo de Tegucigalpa (Rogers, R.D. y E.A. O'Conner, 1993) correspondiente hasta el límite de la línea punteada en la figura del contorno (a). Es aparente la correlación entre las anomalías de Bouguer y la geología, con un contraste máximo de 20 mgals entre la formación Krc (Río Chiquito) y las formaciones del Grupo Padre Miguel (Tpm, Tcg, Tep) dominada por tobas y sedimentos piroclásticos de baja densidad cuya señal gravitacional va disminuyendo suavemente hacia el occidente de la ciudad. 


\subsection{VALLE DE SAN JUAN DE FLORES (CANTARRANAS).}

El Valle de San Juan de Flores, ubicado aproximadamente a $26 \mathrm{Km}$ en dirección Noreste de la ciudad de Tegucigalpa, es un ejemplo de una zona de fractura intraarco (intra arc-rifts) con orientación Nor-Oeste a Sur-Este que aparecen en el bloque Chortís en la región central de Honduras (Rodgers, D. et. al.,2007). El corte del perfil sobre el aluvión tiene una longitud de $5 \mathrm{~km}$ aproximadamente, con estaciones afuera del valle en ambos extremos sobre la formación Cantarranas $(\mathrm{Ka}-\mathrm{c})$ que lo rodea, la cual está compuesta de calizas, limonitas, areniscas y lutitas calcáreas (IGN, 1970).

Las correcciones de topografía que se muestran en la Figura 11c varían entre 2.0 y 3.0 mgals en la mitad Sur del valle (estaciones C8 a C5) que está más próxima a las elevaciones topográficas (Figura 11b) La segunda mitad del perfil, (C4 a C1) más próxima al Valle de Talanga, tiene correcciones entre 1.0 y 2.0 mgals. Esta diferencia entre las dos mitades del perfil producirá una ligera subestimación de la profundidad del aluvión en los puntos más cercanos a las montañas, aunque este efecto es imposible cuantificar.

En la Figura 12 aparecen los gráficos de altura elipsoidal, anomalías de bouguer, y gravedad residual para este perfil. Debido a que nuestro objetivo es explorar la topografía interna del valle (superficie de contacto entre el aluvión y el lecho rocoso), nos interesa escoger un nivel de gravedad regional isostática que produzca valores de gravedad residual negativos en los puntos que están sobre el aluvión.

Este criterio es consistente con el hecho de que el aluvión es menos denso que la roca circundante, exhibiendo un contraste de densidad negativo con respecto a su entorno. Los valores de las anomalías de Bouguer de la Figura 12b reflejan este defecto de masa en la depresión observada entre las estaciones C8 y C12 ubicadas en la zona de contacto entre el aluvión y la formación de Cantarranas. La línea dibujada entre esas estaciones servirá como valor de referencia regional y la diferencia entre las anomalías de Bouguer y los valores producidos por la ecuación de esa recta producen la Figura 12c, de gravedad residual.

Para interpretar nuestras observaciones de gravedad residual, aplicamos un modelo físico y matemático a la cuenca sedimentaria y así predecir sus efectos en el campo de gravedad residual. El programa GRAVMAG (Burger H. et al., 2006) implementa un método numérico de integrales de línea debido a Hubbert (1959), y adaptado para uso en computador por Talwani, Worzel y Landsman (1959) que calcula y suma los efectos gravitacionales en un punto del espacio debidos a 
cuerpos sólidos bidimensionales y tridimensionales de densidad homogénea, discretizados en celdas. Este programa permite construir modelos geológicos en los cuales se especifica la forma poligonal del objeto en dos dimensiones, así como el contraste de densidad con su entorno, y calcula curvas de gravedad residual teóricas.

El mayor contraste de densidad posible para un modelo físico de cuenca aluvial ocurre si asumimos un valor de densidad típica del aluvión de $2.00 \mathrm{~g} / \mathrm{cm}^{3}$ y una densidad de $2.67 \mathrm{~g} / \mathrm{cm}^{3}$ para un lecho rocoso metamórfico, resultando en un contraste de densidad de $-0.67 \mathrm{~g} / \mathrm{cm}^{3}$.

Desconocemos el tipo de rocas que yacen bajo el aluvión, pero sabemos que gran parte del entorno superficial del valle y posiblemente hasta ciertas profundidades está compuesto de rocas calizas y areniscas de la formación Cantarranas, con una densidad menor a $2.67 \mathrm{~g} / \mathrm{cm}^{3}$. Por esta razón asumimos un contraste de densidad de $-0.4 \mathrm{~g} / \mathrm{cm}^{3}$, siguiendo el valor aplicado anteriormente por Al-Zadjali, I. y Stierman, D. (1996) en el valle de Comayagua.

Ejecutamos GRAVMAG asumiendo un contraste de densidad de $-0.4 \mathrm{~g} / \mathrm{cm}^{3}$, modificando progresivamente la forma del cuerpo de aluvión de manera que la gravedad residual teórica calculada se ajuste a la gravedad residual observada en la Figura 12c.

El modelo en dos dimensiones obtenido para la topografía interna de la cuenca sedimentaria del Valle de Cantarranas se aprecia en la Figura 13, con forma ligeramente irregular y profundidades máximas de alrededor de 130 metros. El muestreo de la señal gravitacional, de una muestra por km, impide observar irregularidades en la topografía interna de longitudes de onda inferiores a $2 \mathrm{~km}$.

Al reproducir los cálculos asumiendo un basamento de roca metamórfica cristalina con un contraste de densidad de $-0.67 \mathrm{~g} / \mathrm{cm}^{3}$, obtuvimos un modelo de cuenca con la misma geometría de la Figura 13 pero resulta en una profundidad máxima de 70 $\mathrm{m}$, que representa el valor mínimo posible para la profundidad máxima de la cuenca. 


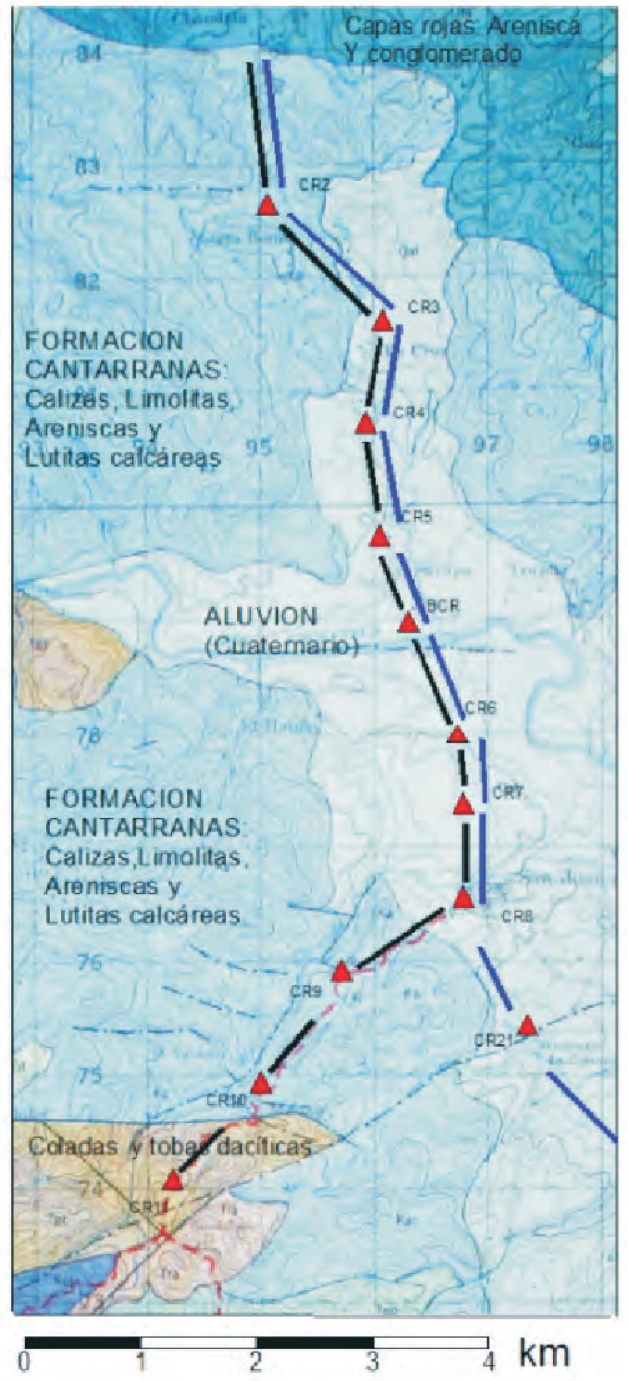

a. Geologia del Valle de Cantarranas Perfil C1 - C11

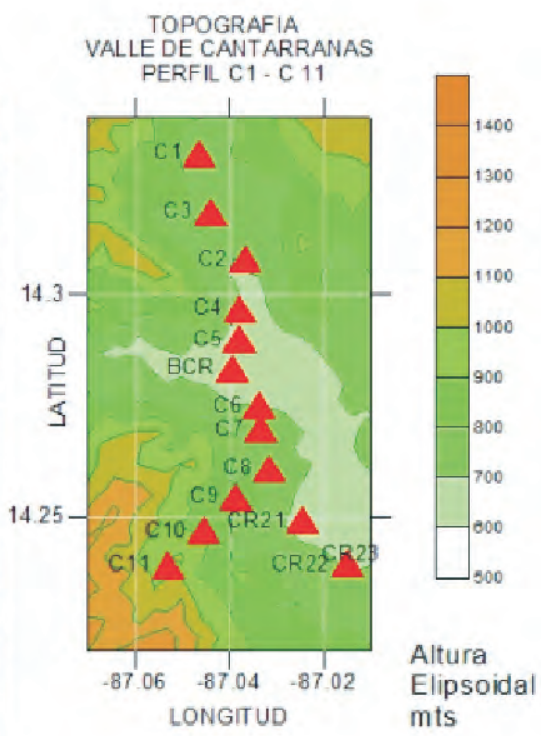

b

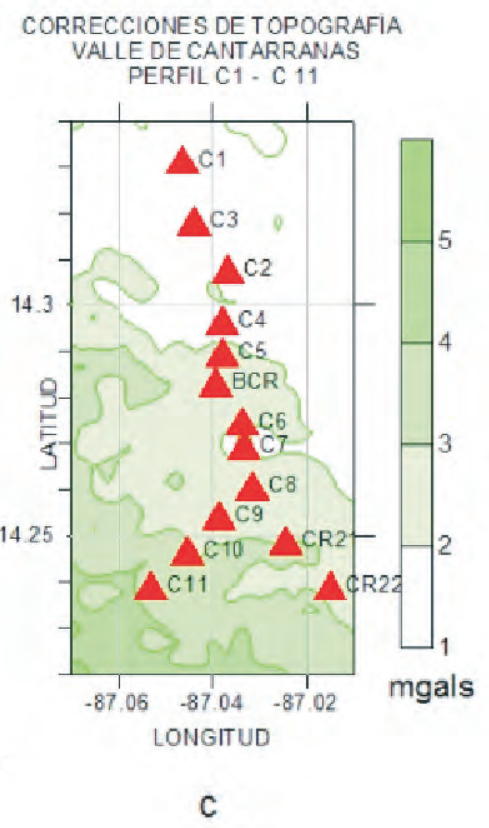

Figura 11. (a) Geología del Valle de San Juan de Flores (Cantarranas), según IGN(1970). (b) y (c) Modelo de elevación digital y correcciones de topografía calculadas con HAMXYZ2. 
Tabla 7. Datos y ajustes experimentales para el perfil CR11-CR1 Valle de Cantarranas

\begin{tabular}{|c|c|c|c|c|c|}
\hline Estación & $\begin{array}{c}\text { ÄG } \\
\text { Gravímetro } \\
\text { (MGAL) }\end{array}$ & Error & $\begin{array}{c}\text { RMS } \\
\text { Error }\end{array}$ & $\begin{array}{c}\text { Corrección } \\
\text { de Marea }\end{array}$ & $\begin{array}{c}\text { Deriva } \\
\text { Instru- } \\
\text { mental }\end{array}$ \\
\hline CR11 & 1785.421 & 0.003 & 0.098 & -0.075 & -0.008 \\
\hline CR10 & 1810.083 & 0.009 & 0.072 & -0.070 & -0.007 \\
\hline CR9 & 1823.845 & 0.009 & 0.055 & -0.065 & -0.006 \\
\hline CR8 & 1842.197 & 0.005 & 0.036 & -0.058 & -0.004 \\
CR7 & 1841.316 & 0.002 & 0.061 & -0.050 & -0.003 \\
\hline CR6 & 1847.699 & 0.002 & 0.044 & -0.043 & -0.001 \\
\hline CR4 & 1851.336 & 0.002 & 0.047 & -0.020 & 0.012 \\
\hline CR3 & 1849.095 & 0.005 & 0.029 & -0.012 & 0.010 \\
\hline CR2 & 1850.152 & 0.004 & 0.02 & 0.004 & 0.008 \\
\hline CR1 & 1845.860 & 0.004 & 0.028 & 0.005 & 0.005 \\
\hline
\end{tabular}

Tabla 8. Correcciones gravimétricas y Anomalía de Bouger para el perfil CR11CR1. *Medida del gravímetro corregida por efecto de marea y deriva instrumental

\begin{tabular}{|c|c|c|c|c|c|c|c|c|c|c|c|}
\hline $\begin{array}{l}\text { Esta- } \\
\text { ción }\end{array}$ & $\begin{array}{l}\text { Latitud } \\
\text { (grados) }\end{array}$ & $\begin{array}{l}\text { Longitud } \\
\text { (grados) }\end{array}$ & $\begin{array}{c}\text { Altura } \\
\text { Ortamé- } \\
\text { trica (M) }\end{array}$ & $\begin{array}{l}\text { Altura } \\
\text { Elipsoidal } \\
\text { (M) }\end{array}$ & $\begin{array}{l}\triangle G^{*} \\
(\text { MGAL) }\end{array}$ & $\begin{array}{l}\text { Gravedad } \\
\text { Absoluta } \\
\text { (MGAL) }\end{array}$ & $\begin{array}{c}\text { Correccion } \\
\text { por Latitud } \\
\text { (MGAL) }\end{array}$ & $\begin{array}{c}\text { Corrección } \\
\text { de aire llbre } \\
\text { (MGAL) }\end{array}$ & $\begin{array}{l}\text { Corrección } \\
\text { do Bouger } \\
\text { (MGAL) }\end{array}$ & $\begin{array}{c}\text { Comección } \\
\text { Topografica } \\
\text { (MGAL) }\end{array}$ & $\begin{array}{l}\text { Anomalia de } \\
\text { Bouger } \\
\text { (MGAL) }\end{array}$ \\
\hline CRO & 1.2468324 & -87.0454107 & 877.14 & 883.64 & 1810.080 & 978109.060 & 978344.627 & 272.528 & 98.198 & 2.951 & 58.287 \\
\hline CR9 & 14.2545219 & -87.0386296 & 814,74 & 821.21 & 1823.851 & 978122.821 & 978344.958 & 253.273 & 97.213 & 2.450 & 52,027 \\
\hline CR8 & 14.2608348 & -87.0315122 & 723.24 & 729.89 & 1842.201 & 978141.171 & 978345.230 & 225.047 & 80.869 & 2.481 & 57.501 \\
\hline CR7 & 14.269657 & -87.0333118 & 729.09 & 735.52 & 1841.319 & 978140.289 & 978345.610 & 226.844 & 81.624 & 2138 & 57.963 \\
\hline CR2 & 11.3185087 & -87.0440088 & 735.8 & 741.80 & 1850.74 & $9781<9.131$ & 978347717 & 228.782 & 82339 & 1775 & 60.368 \\
\hline CR1 & 14.3316691 & .87 .0464241 & 766.92 & 773.21 & 1845.855 & 978144.BA2 & 978348.286 & 238.469 & 85.859 & 1.431 & 49,403 \\
\hline
\end{tabular}
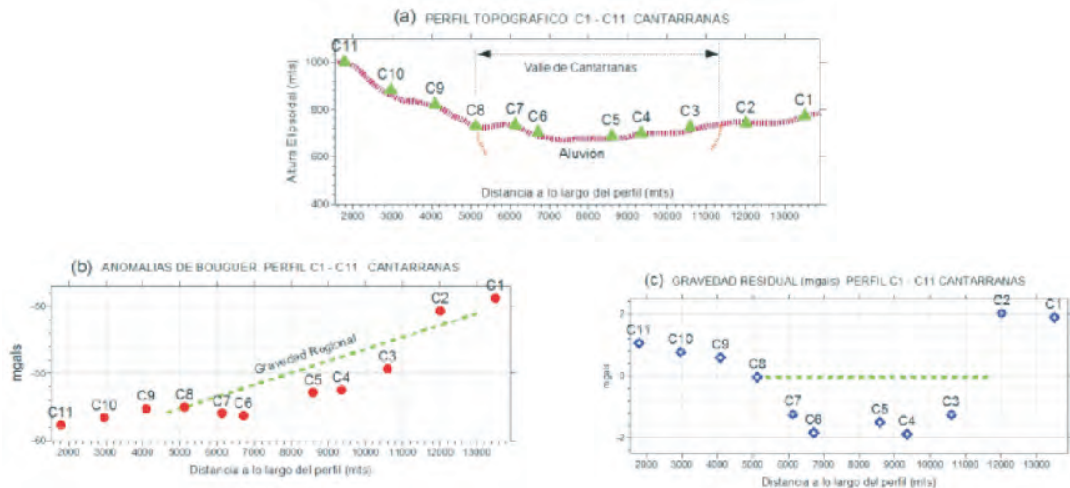

Figura 12. Topografía, anomalías de bouguer y gravedad reducida para el perfil C1-C11 del Valle de San Juan de Flores (Cantarranas). La línea verde indica la definición de gravedad regional para este problema, escogida de manera que la gravedad residual sea negativa donde haya un contraste de densidad negativo, entre las estaciones C8 y C2 de (a). 
MODELO GEOLOGICO PARA EL PERFIL CR1 - CR11 CANTARRANAS
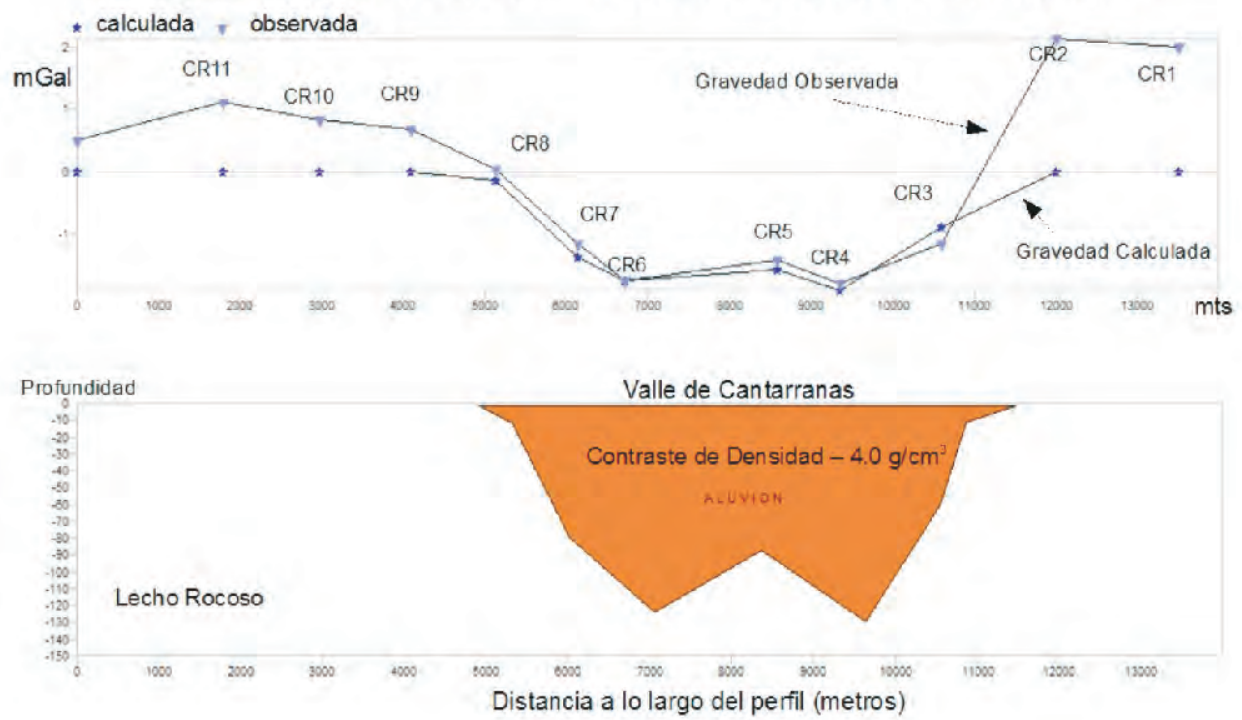

Figura 13. Modelo geológico de la cuenca sedimentaria para el perfil CR1-CR11 del Valle de Cantarranas. La profundidad máxima del valle oscila entre 80 y 130 m aproximadamente.

\subsection{VALLE DE MOROCELÍ}

El Valle de Morocelí es la continuación del Valle de Cantarranas, siguiendo la misma fractura intra arco con orientación NO-SE que se ensancha en dirección SE hasta la comunidad de Ojo de Agua. El relleno del valle consiste, en su flanco nor-occidental, de conos y abanicos aluviales de pie de monte (Qaf), y el resto, de aluvión reciente de inundación del río Choluteca. Rodean la cuenca terrazas de arena grava y arcilla roja (Tcal) y la formación Tpm de tobas e ignimbritas riolíticas (IGN,1996).

La Figura 14a muestra la sección correspondiente al mapa geológico del área de estudio (IGN, 1996) y la localización de las estaciones del perfil CR312-C8 con una longitud total de $37 \mathrm{~km}$. que cruza el aluvión del valle de Morocelí en dirección de Sur-Este a Nor-Oeste.

Las estaciones del perfil están espaciadas a intervalos de aproximadamente $2 \mathrm{~km}$. Los valores y ajustes de los datos experimentales y las correcciones gravimétricas y anomalías de Bouguer para esos puntos se muestran en las Tablas 9 y 10, 
respectivamente. En la Figura 14b las correcciones de topografía se muestran como mapa de contorno y su valor es relativamente uniforme en todo el valle, con efectos menores hacia el Sur Este, lejos de las elevaciones topográficas de la Figura 14c. En la Figura 15 aparecen los gráficos de altura elipsoidal, anomalías de bouguer, y gravedad residual para este perfil, que tiene la estación C8 en común con el perfil anterior.

Dicha estación está localizada en el contacto entre aluvión y la formación de Cantarranas, Kac. Siguiendo los criterios expuestos en la sección anterior, escogimos un nivel de gravedad residual consistente con el punto común C8 del perfil C1-C11, de manera que el nivel cero es el mismo para ambos perfiles.

Los resultados para este perfil se ilustran en la Figura 16. Ejecutamos GRAVMAG con un contraste de densidad de $-0.4 \mathrm{~g} / \mathrm{cm} 3$ entre el lecho rocoso y el aluvión. Además ajustamos el modelo geométrico de la cuenca sedimentaria para lograr el mejor ajuste de la curva teórica con la curva de gravedad residual observada. Para este perfil, tenemos una topografía interna marcadamente irregular con afloramientos del lecho rocoso que dividen la cuenca sedimentaria en tres secciones.

El valor cero para CR35 en la curva de gravedad residual coincide con un afloramiento de la formación Tpm en la vecindad de dicha estación (Figura 14a). Este afloramiento rocoso en CR35 divide al valle en dos subcuencas: La primera entre las estaciones CR311 y CR35 tiene una profundidad máxima de 400m, es poco relativamente; y de topografía relativamente suave; La segunda, entre CR35 y CR24, es de fondo marcadamente irregular y de mayor profundidad, oscilando entre 1000,300 y 1,400 metros.

Los valores positivos de gravedad residual en CR22 CR23 y CR24 están asociados al contacto del aluvión al borde del valle. No existe carta geológica disponible para esazona. 


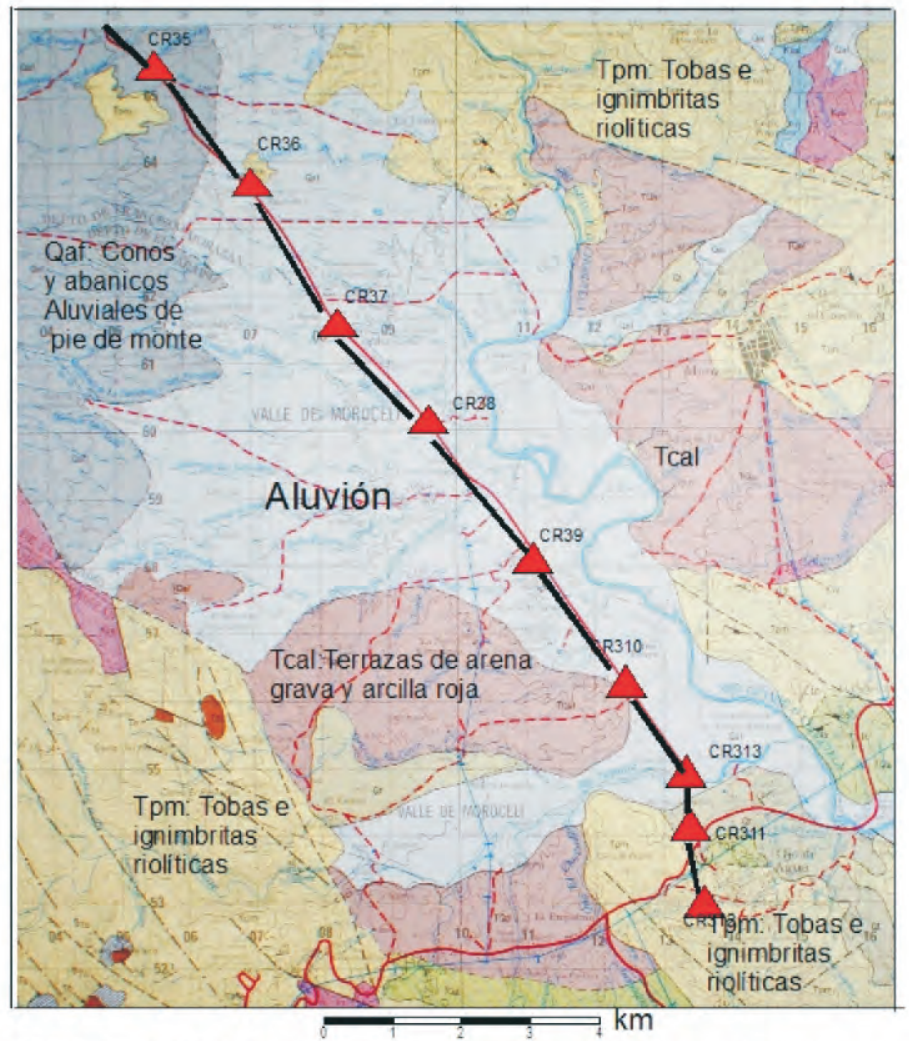

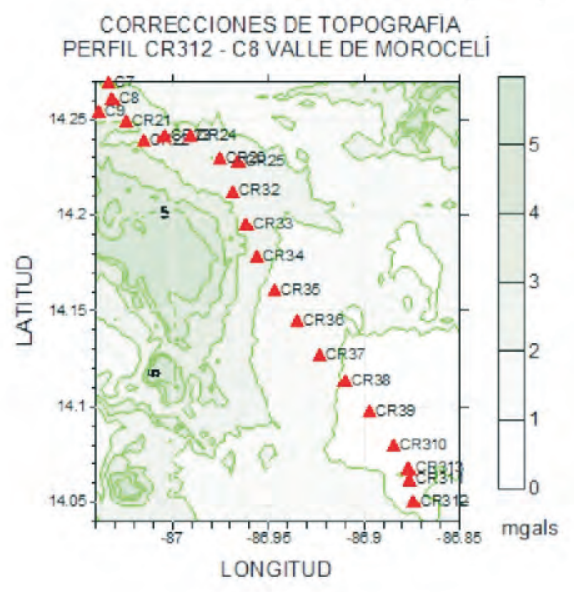

b a TOPOGRAFIA DEL VAUE DE MOROCEU

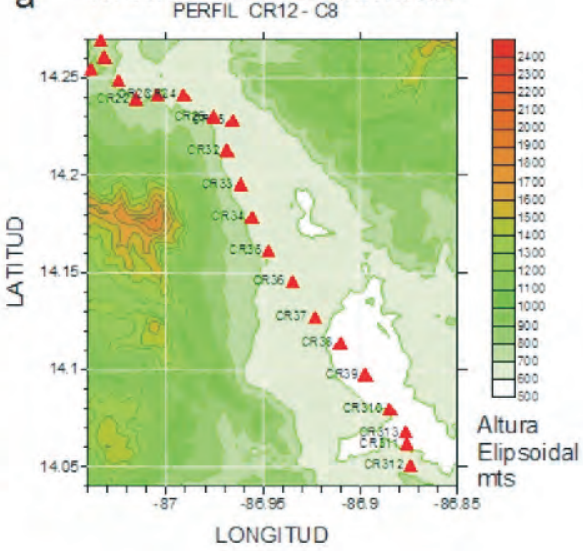

C

Figura 14. (a) Geología del Valle de Morocelí, según IGN(1996). (b) y (c) Modelo de elevación digital y correcciones de topografía calculadas con HAMXYZ2. 
Tabla 9. Datos y ajustes experimentales para el perfil CR312-CR8

\begin{tabular}{|c|c|c|c|c|c|}
\hline Estación & $\begin{array}{c}\text { ÄG } \\
\text { Gravímetro } \\
\text { (MGAL) }\end{array}$ & Error & $\begin{array}{c}\text { RMS } \\
\text { Error }\end{array}$ & $\begin{array}{c}\text { Corrección } \\
\text { de Marea }\end{array}$ & $\begin{array}{c}\text { Deriva } \\
\text { Instru- } \\
\text { mental }\end{array}$ \\
\hline CR312 & 1846.239 & 0.005 & 0.065 & 0.029 & -0.018 \\
\hline CR311 & 1854.772 & & & & -0.016 \\
\hline CR313 & 1857.673 & 0.004 & 0.062 & 0.040 & -0.020 \\
\hline CR310 & 1854.674 & 0.005 & 0.037 & 0.001 & -0.013 \\
\hline CR39 & 1854.900 & 0.004 & 0.07 & -0.007 & -0.012 \\
\hline CR38 & 1852.442 & 0.005 & 0.078 & -0.017 & -0.011 \\
\hline CR37 & 1848.585 & & & & -0.010 \\
\hline CR36 & 1845.837 & 0.004 & 0.019 & -0.034 & -0.008 \\
\hline CR35 & 1844.659 & 0.001 & 0.022 & -0.044 & -0.007 \\
\hline CR34 & 1841.735 & 0.003 & 0.017 & -0.058 & -0.005 \\
\hline CR33 & 1837.276 & 0.006 & 0.048 & -0.068 & -0.003 \\
\hline CR32 & 1841.825 & 0.006 & 0.026 & -0.077 & -0.002 \\
\hline CR26 & 1854.774 & 0.003 & 0.096 & -0.170 & 0.003 \\
\hline CR25 & 1845.541 & 0.005 & 0.017 & -0.167 & 0.002 \\
\hline CR22 & 1845.699 & 0.002 & 0.036 & -0.146 & 0.000 \\
\hline CR23 & 1843.734 & 0.006 & 0.031 & -0.155 & 0.001 \\
\hline CR24 & 1854.791 & 0.006 & 0.094 & -0.162 & 0.002 \\
\hline CR21 & 1847.055 & 0.002 & 0.025 & -0.132 & 0.000 \\
\hline CR8 & 1842.197 & 0.005 & 0.036 & -0.058 & -0.004 \\
\hline
\end{tabular}

Tabla 10. Correcciones gravimétricas y Anomalía de Bouger para el perfil CR312-CR8. *Medida del gravímetro corregida por efecto de marea y deriva instrumental

\begin{tabular}{|c|c|c|c|c|c|c|c|c|c|c|c|}
\hline $\begin{array}{l}\text { Esta- } \\
\text { ción }\end{array}$ & $\begin{array}{l}\text { Latitud } \\
\text { (grados) }\end{array}$ & $\begin{array}{l}\text { Longitud } \\
\text { (grados) }\end{array}$ & $\begin{array}{l}\text { Altura } \\
\text { Ortomé- } \\
\text { trica (M) }\end{array}$ & $\begin{array}{l}\text { Altura } \\
\text { Elipsoidal } \\
\text { (M) }\end{array}$ & $\begin{array}{c}\Delta G^{+} \\
(M G A L)\end{array}$ & $\begin{array}{l}\text { Gravedad } \\
\text { Absoluta } \\
\text { (MGAL) }\end{array}$ & $\begin{array}{l}\text { Correcclón } \\
\text { por Latitud } \\
\text { (MGAL) }\end{array}$ & $\begin{array}{c}\text { Correccion } \\
\text { de aire libre } \\
\text { (MGAL) }\end{array}$ & $\begin{array}{l}\text { Correceión } \\
\text { de Bouger } \\
\text { (MGAL) }\end{array}$ & $\begin{array}{c}\text { Carrocción } \\
\text { Topográfica } \\
\text { (MGAL) }\end{array}$ & $\begin{array}{l}\text { Anomalla de } \\
\text { Bouger } \\
\text { (MGAL) }\end{array}$ \\
\hline CR312 & 410505205 & -85.6740594 & 641.44 & 6.7 .93 & 1846.257 & 978145.001 & 978336.237 & 199.828 & 71.811 & 0.902 & -62.317 \\
\hline CR311 & 14.0619687 & $-86,8760669$ & 592.88 & 599.37 & 1854.788 & 978153.532 & 978336.723 & 184.851 & 66.375 & 0.903 & -63.812 \\
\hline CR313 & (1) 0678400 & -85.8765548 & 571.54 & 578.13 & 1857683 & 978156.437 & 978336.973 & 178.299 & 63997 & 0.969 & -65.265 \\
\hline CR310 & 14.0497323 & -86.8848002 & 581.23 & 587.72 & 1854.687 & 978153.431 & 978337.479 & 181.257 & 65.070 & 0.843 & -67.018 \\
\hline CA39 & 140375805 & -86.8971574 & 583.91 & 590.40 & $185 \subset 912$ & 978153.656 & 978338.239 & 182.085 & 65.371 & 0.872 & -66.995 \\
\hline CR38 & 14.1135373 & -86.9102089 & 588.50 & 594.98 & 1852.453 & 978151.197 & 978338.919 & 183.498 & 65.884 & 0.974 & -69.134 \\
\hline CR37 & 18.127 .1315 & -88.9234080 & E13.58 & 620.07 & 1848.595 & 978147.339 & 978339.498 & 191.235 & 58.692 & 1,092 & -68.525 \\
\hline CR36 & 14.145 .1308 & -86.9349079 & 654.18 & 660.67 & 1845.845 & 978144.589 & 978340.267 & 203.758 & 73.238 & 1.270 & 63.888 \\
\hline CR35 & 14.1608193 & .86 .9470874 & 673.17 & 679.65 & $184<686$ & 978143.410 & 978340.938 & 209.612 & 75353 & 1.668 & -61.612 \\
\hline CR34 & 14.1783178 & -86.9559995 & 667.28 & 673.75 & 1841.740 & 978140.484 & 978341.687 & 207.793 & 74.704 & 2.434 & -65.679 \\
\hline CR33 & 14.1954248 & 86.9616205 & 654.86 & 661.33 & 1837279 & 978136.023 & 973342.420 & 203.960 & 73314 & 2.870 & 72.381 \\
\hline CR32 & 14.2126516 & -86.9685173 & 665.97 & 672.41 & 1841.827 & 978140.571 & 978343.158 & 207.380 & 74.557 & 2.472 & -67.293 \\
\hline CR2E & 14.2281023 & .86 .9658349 & 574.20 & 680.63 & 185477 & 978153.661 & 978343.822 & 209.914 & 75.478 & 2.131 & 53.595 \\
\hline CR25 & 14.2301058 & -88.9754587 & 613.31 & 619.73 & 1845.539 . & 978144.429 & 978343.908 & 191.130 & 68.661 & 2.411 & .74 .600 \\
\hline CR22: & 112392503 & -870151350 & 692.49 & 698.96 & 1815.699 & 978144.589 & 973344.301 & 215559 & 7.526 & 2.982 & 58.688 \\
\hline CR23 & 14,2414623 & -87.0043654 & 712.00 & 718.46 & 1843.733 & 978142.623 & 978344.396 & 221.580 & 79.710 & 2.098 & -57.805 \\
\hline CR24 & 142415358 & -86.9909191 & 553.15 & 658.59 & 1854769 & 979153.579 & 978614.398 & 20.3 .424 & 73.122 & 2.174 & -58.245 \\
\hline CR21 & 14.2490441 & -87.0245449 & 679.14 & 685.61 & 1847.055 & 978145.945 & 978344.722 & 211.450 & 76.032 & 3.059 & -60.301 \\
\hline CRB & 742608348 & -87.0315122 & 723.24 & 729.69 & 1842204 & 978141171 & 978345.230 & 225.047 & 30.950 & 2.481 & 57.501 \\
\hline
\end{tabular}


(a) PERFIL TOPOGRAFICO CR312 - C8 MOROCELI

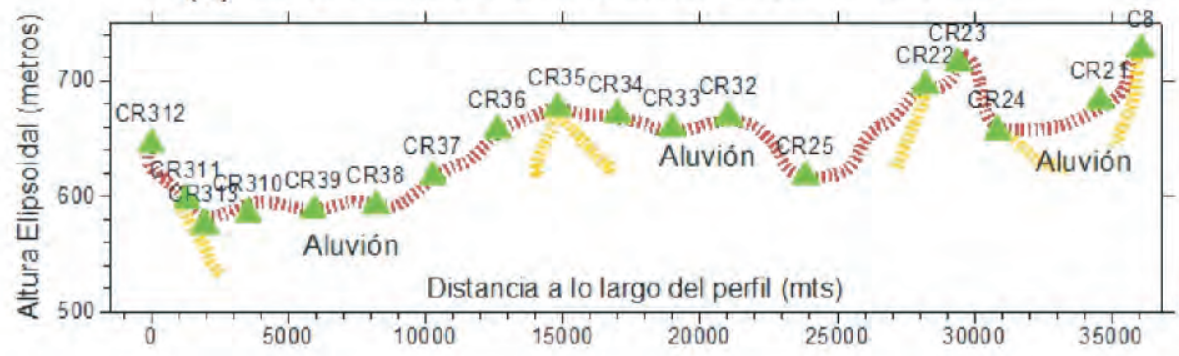

(b) ANOMALÍAS DE BOUGUER PERFIL C312 - C8 MOROCELÍ

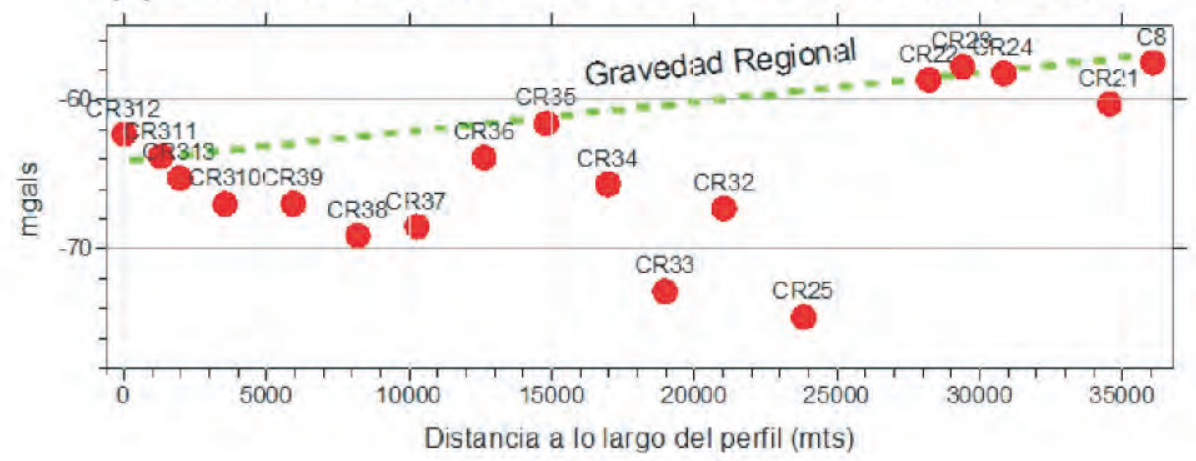

(c) GRAVEDAD RESIDUAL (mgals) PERFIL CR311 - C8 MOROCELÍ

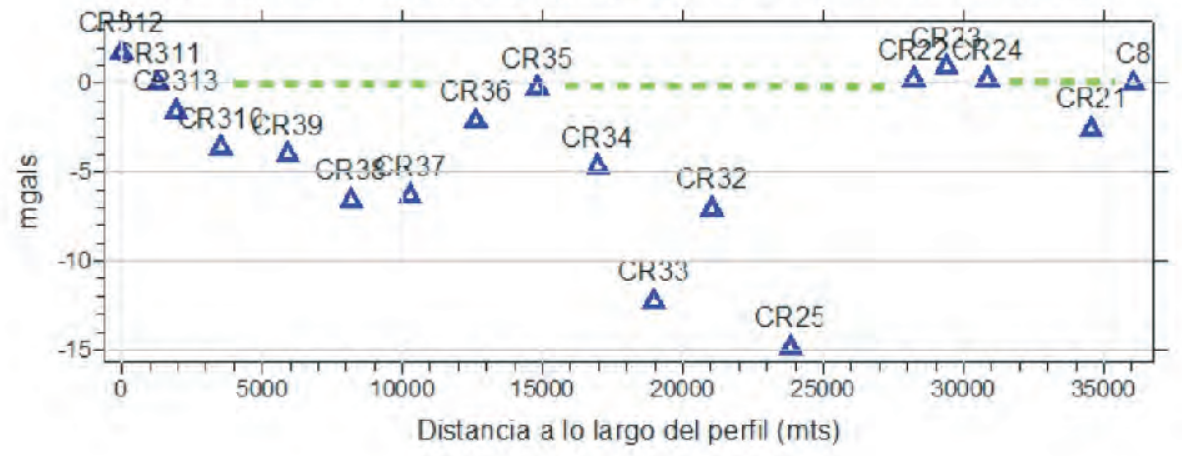

Figura 15. Topografía, anomalías de bouguer y gravedad reducida para el perfil C312C8 del Valle de Morocelí. La línea verde indica la definición de gravedad regional para este problema, escogida para que la gravedad residual sea negativa donde haya un contraste de densidad negativo, entre las estaciones C313 y C8. Esta elección es consistente además con el afloramiento de roca en CR35 de (a) 


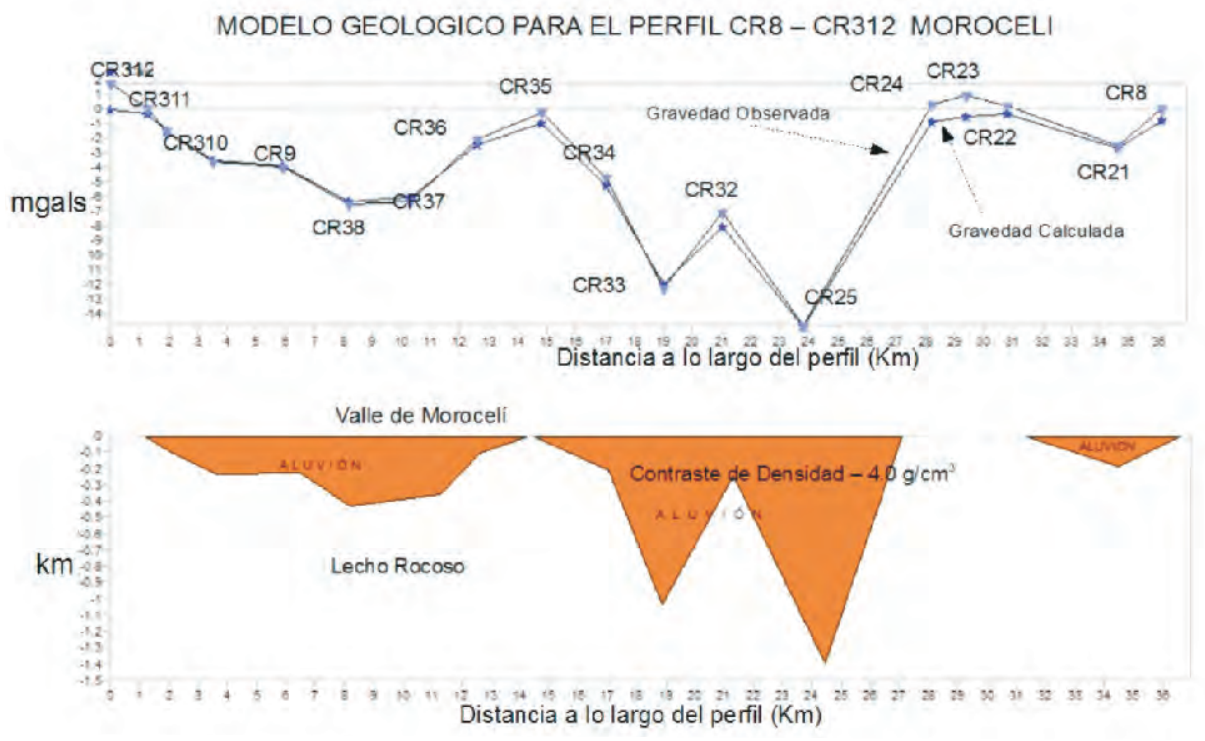

Figura 16. Modelo geológico de la cuenca sedimentaria para el perfil CR312-CR8 del Valle de Morocelí.

\subsection{Valle de El Zamorano.}

La información geológica del Valle del Zamorano se limita a la obtenida del mapa geológico general de Honduras, (Elvir, 1997) ya que no se cuenta con una carta geológica detallada de la zona. Del mapa geológico general (Figura 17.a) se observa que la zona del valle del Zamorano se encuentra localizada entre depósitos volcánicos del cuaternario compuestos de basalto, andesita, flujos piroclásticos y rocas volcánicas del terciario pertenecientes al grupo Matagalpa y Padre Miguel. Los depósitos sedimentarios del Valle corresponden a aluvión del cuaternario (Elvir, 1997). En cuanto a la topografía de la zona de estudios, los valores de altura elipsoidal para la localización de las estaciones, oscila entre los $1185.04 \mathrm{~m}$ y $751.41 \mathrm{~m}$ (tablas 10,11,12 y 13 y figura 17b). Las correcciones por topografía obtenidas para la mayor parte de las estaciones se consideran relativamente altas (entre 1.5 y 2.5 mgal), este resultado se puede atribuir a que el Valle es relativamente pequeño, por lo que la influencia de las montañas circundantes se hace más evidente. Sin embargo, al observar el mapa de correcciones topográficas (figura 17.c), debido a que la mayor parte de las estaciones se localizan en el Valle, se observa un patrón uniforme, por lo que podemos asumirse que el efecto topográfico no contribuye significativamente al cambio en la tendencia del comportamiento local de los resultados. 


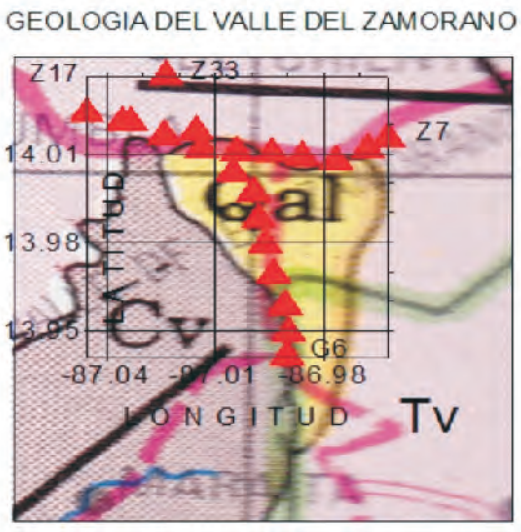

a

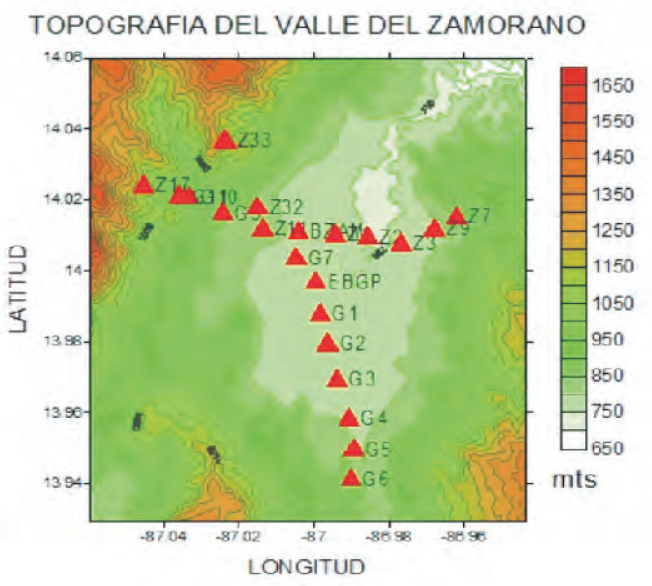

b

\section{CORRECCIONES DE TOPOGRAFIA VALLE DEL ZAMORANO}

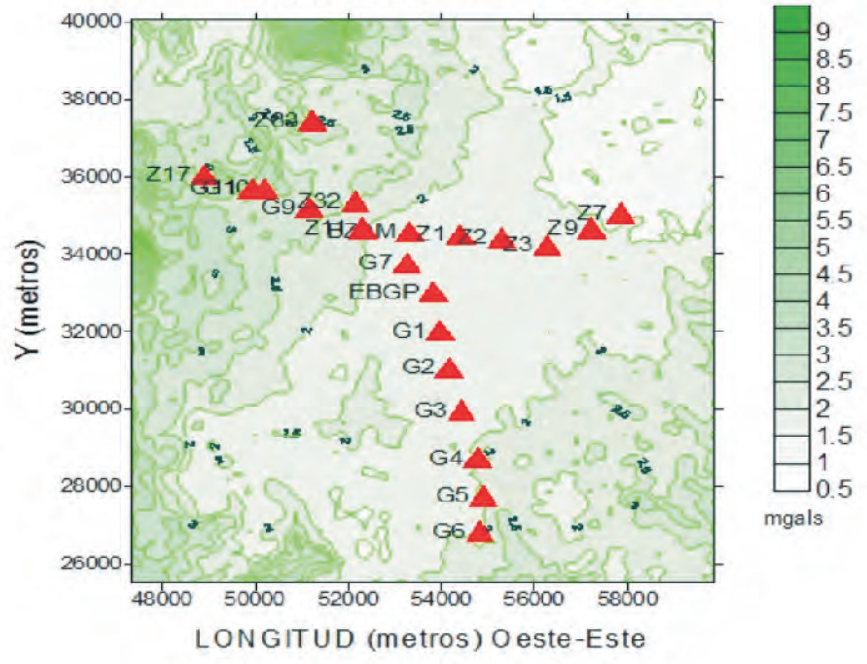

C

Figura 17. a Sección del mapa geológico de Honduras correspondiente a la zona de estudio, Cv: depósitos volcánicos de cuaternario, Tv: Rocas volcánicas del terciario y Qa: Aluvión de cuaternario. Sobre el mapa geológico de la zona se indica con triángulos la localización de las estaciones gravimétricas. b Mapa en el que se muestra la topografía de la zona, las montañas más altas circundantes presentan altura elipsoidal de alrededor de 1180 m c Correcciones de Topografía, valores mínimos alrededor de 1.5 mgals, las

estaciones localizadas a los largo del Valle presentan correcciones uniformes de topografía. 
Tabla 10. Datos y ajustes experimentales para el perfil Z17-Z7

\begin{tabular}{|c|c|c|c|c|c|}
\hline Estación & $\begin{array}{c}\text { ÄG } \\
\text { Gravímetro } \\
\text { (MGAL) }\end{array}$ & Error & $\begin{array}{c}\text { RMS } \\
\text { Error }\end{array}$ & $\begin{array}{c}\text { Corrección } \\
\text { de Marea }\end{array}$ & $\begin{array}{c}\text { Deriva } \\
\text { Instru- } \\
\text { mental }\end{array}$ \\
\hline Z17 & 1748.072 & 0.012 & 0.026 & -0.067 & -0.328 \\
\hline G11 & 1784.811 & 0.002 & 0.035 & -0.122 & -0.004 \\
\hline G10 & 1789.521 & 0.003 & 0.019 & -0.127 & -0.003 \\
\hline G9 & 1799.893 & 0.002 & 0.027 & -0.129 & -0.002 \\
\hline Z11 & 1804.925 & 0.004 & 0.026 & -0.107 & -0.060 \\
\hline BZAM & 1808.623 & 0.016 & 0.092 & -0.108 & \\
\hline Z1 & 1814.934 & 0.006 & 0.027 & -0.121 & 0.017 \\
\hline Z2 & 1821.077 & 0.003 & 0.093 & -0.133 & 0.035 \\
\hline Z3 & 1820.860 & 0.005 & 0.08 & -0139 & 0.044 \\
\hline Z4 & 1816.723 & 0.004 & 0.021 & -0.144 & 0.055 \\
\hline Z7 & 1809.523 & 0.005 & 0.08 & -0.147 & -0.030 \\
\hline
\end{tabular}

Tabla 11. Correcciones gravimétricas y Anomalía de Bouger para el perfil Z17-Z7

\begin{tabular}{|c|c|c|c|c|c|c|c|c|c|c|c|}
\hline $\begin{array}{l}\text { Esta- } \\
\text { clón }\end{array}$ & $\begin{array}{l}\text { Latitud } \\
\text { (grados) }\end{array}$ & $\begin{array}{l}\text { Longitud } \\
\text { (grados) }\end{array}$ & $\begin{array}{l}\text { Altura } \\
\text { Ortomé- } \\
\text { trica (M) }\end{array}$ & $\begin{array}{l}\text { Altura } \\
\text { Elipsoidal } \\
\text { (M) }\end{array}$ & $\begin{array}{c}\Delta G^{*} \\
\text { (MGAL) }\end{array}$ & $\begin{array}{l}\text { Gravedad } \\
\text { Absoluta } \\
\text { (MGAL) }\end{array}$ & $\begin{array}{l}\text { Correccion } \\
\text { por Latitud } \\
\text { (MGAL) }\end{array}$ & $\begin{array}{l}\text { Correcclón } \\
\text { de aire libre } \\
\text { (MGAL) }\end{array}$ & $\begin{array}{l}\text { Corrección } \\
\text { de Bouger } \\
\text { (MGAL) }\end{array}$ & $\begin{array}{c}\text { Corrección } \\
\text { Topográfica } \\
\text { (MGAL) }\end{array}$ & $\begin{array}{l}\text { Anomalía de } \\
\text { Bouger } \\
\text { (MGAL) }\end{array}$ \\
\hline Z11 & 14.0206964 & .87 .0356241 & 941.92 & 948.55 & 1784.815 & 978084.648 & 878334.972 & 282.547 & 105451 & 3714 & -59.513 \\
\hline 610 & 40206517 & 87,0331940 & $9 \cdot 7.74$ & 92437 & 1789.524 & 978089.357 & 978334970 & 285.089 & 102743 & 3.941 & 59327 \\
\hline 69 & 14.0161306 & .87 .0241905 & 850.79 & 857.42 & 1799.895 & 978099.728 & 978334.778 & 264.440 & 95.248 & 3.059 & -62.799 \\
\hline Z11 & 14,0115330 & 87.0136004 & 809.82 & 816.44 & 1804.985 & 978104.281 & 978334.583 & 251.801 & 90.662 & 2212 & -86951 \\
\hline 23 & 14.0074526 & .86 .9766600 & 755.05 & 761.64 & 1820816 & 978120.113 & 978334.411 & 254.900 & 61.531 & 1756 & -62773 \\
\hline 24 & 14.0034861 & -86.9712430 & 781.42 & 788.00 & 1816,668 & 978115.965 & 978334.243 & 243.028 & 87.482 & 1.672 & -61.030 \\
\hline 27 & 14.0149680 & .56 .9619963 & 835.14 & 841.71 & 1809.553 & 578108.751 & 978334.729 & 259596 & 93.496 & 1384 & -58.495 \\
\hline
\end{tabular}

Tabla 12. Datos y ajustes experimentales para el perfil Z33-G6

\begin{tabular}{|c|c|c|c|c|c|}
\hline Estación & $\begin{array}{c}\ddot{A G G} \\
\text { Gravímetro } \\
\text { (MGAL) }\end{array}$ & Error & $\begin{array}{l}\text { RMS } \\
\text { Error }\end{array}$ & $\begin{array}{c}\text { Corrección } \\
\text { de Marea }\end{array}$ & $\begin{array}{l}\text { Deriva } \\
\text { Instru- } \\
\text { mental }\end{array}$ \\
\hline Z33 & 1736.379 & 0.009 & 0.026 & -0.111 & 0.023 \\
\hline Z32 & 1805.191 & 0.004 & 0.082 & -0.117 & 0.014 \\
\hline Z11 & 1804.925 & 0.004 & 0.026 & -0.107 & -0.060 \\
\hline G7 & 1808.951 & 0.008 & 0.089 & -0.13 & -0.001 \\
\hline $\mathrm{Bg} 1$ & 1810.438 & 0.004 & 0.07 & -0.057 & \\
\hline G1 & 1811.217 & 0.008 & 0.064 & -0.068 & 0.003 \\
\hline $\mathrm{G} 2$ & 1810.939 & 0.005 & 0.089 & -0.076 & 0.005 \\
\hline G3 & 1809.795 & 0.031 & 0.05 & -0.084 & 0.008 \\
\hline G4 & 1812.707 & 0.003 & 0.031 & -0.097 & 0.012 \\
\hline G5 & 1808.530 & 0.003 & 0.044 & -0.106 & 0.015 \\
\hline G6 & 1796.150 & 0.002 & 0.034 & -0.113 & 0.018 \\
\hline
\end{tabular}


Tabla 13. Correcciones gravimétricas y Anomalías de Bouger para el perfil Z33G6

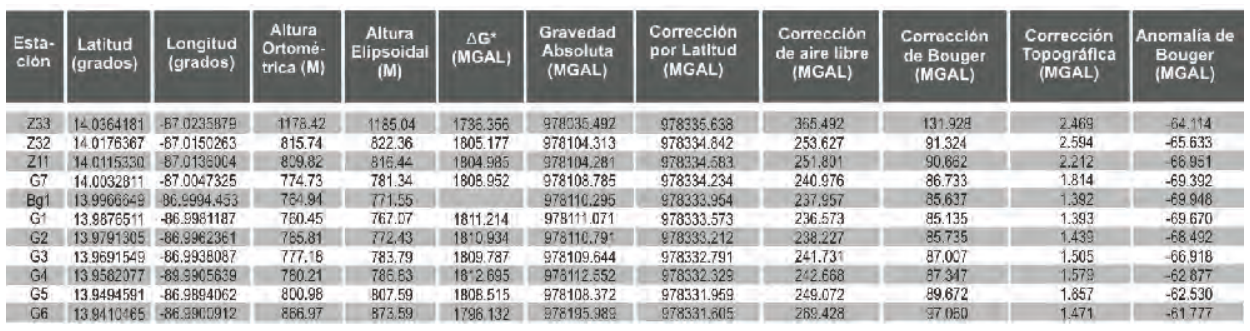

* Medida del gravímetro corregida por efecto de marea y deriva instrumental

Para la interpretación de resultados seleccionamos dos perfiles (Figuras 18a y 18c, tablas 10-13). El perfil Z17-Z7 se extiende transversalmente y el perfil Z33-G6 se extiende longitudinalmente, ambos perfiles tiene una estación en común (Z11). Interpretamos los datos utilizando el método de interpretación cualitativa de suavizado gráfico (Cantos, 1974). A fin de obtener la influencia gravimétrica atribuida a la capa de aluvión presente en la cuenca sedimentaria, supusimos una tendencia regional lineal y removimos esta contribución de las anomalías de Bouguer observadas, siguiendo similares criterios a los utilizados en los perfiles descritos en la sección 4.2.; de esta forma obtuvimos las anomalías residuales atribuidas al aluvión. Para el perfil Z33-G6, los límites de la interfase entre el aluvión y el basamento subyacente son las estaciones $Z 32$ y G4, la línea regional pasa por estos límites y las estaciones entre estos límites presentan anomalías residuales negativas atribuidas al aluvión (Figura 18c.b). Para el perfil Z17-Z7 definimos una línea regional entre las estaciones G9 y G11 en un extremo y Z4 en el otro extremo (Figura 18a.b). Los valores de las anomalías residuales obtenidas en el perfil Z33G6 van desde 2.422 mgal en la zona más alta, hasta $-5.294 \mathrm{mgals}$ en el centro del perfil de forma similar en el perfil Z17-Z7 van desde 8.401 mgals hasta -4.039 mgals, observándose una tendencia decreciente de las anomalías hacia el centro delvalle.

Utilizando el programa GRAVMAG, con el propósito de obtener un modelo geológico del valle a partir de las anomalías gravimétricas, ajustamos los valores de las anomalías residuales obtenidas a un modelo de dos capas (Figuras 18b y 18d) siguiendo los mismos criterios y procedimiento utilizados para modelar los perfiles de la sección 4.2. En el modelo presentado en las figuras 18b y 18d asumimos una densidad fija para cada capa, con un contraste de densidades entre la roca circundante y el aluvión de $-0.4 \mathrm{~g} / \mathrm{cm}^{3}$. De acuerdo a los resultados obtenidos en los perfiles, la profundidad máxima de los sedimentos se observa en la parte central del 
valle y se estima que es alrededor de $350 \mathrm{~m}$. Con el propósito de establecer rangos límites de profundidad, se elaboraron modelos similares a los de las figuras 18b y $18 \mathrm{~d}$, pero suponiendo un contraste de densidad de $-0.67 \mathrm{~g} / \mathrm{cm}^{3}$, que es el valor para basamento granítico subyacente y aluvión. Con este contraste los valores máximos de profundidad observados son un poco superiores a los 200m. De acuerdo a los resultados de estos modelos puede afirmarse que las profundidades máximas del Valle del Zamorano, localizadas en la parte central del mismo, se encuentran en el rango de $350 \mathrm{~m}$ a $200 \mathrm{~m}$. En cuanto a la forma de los depósitos de aluvión, independientemente del contraste de densidad supuesto, el perfil longitudinal muestra un valle en forma de $V$ que es propio de relieves jóvenes.
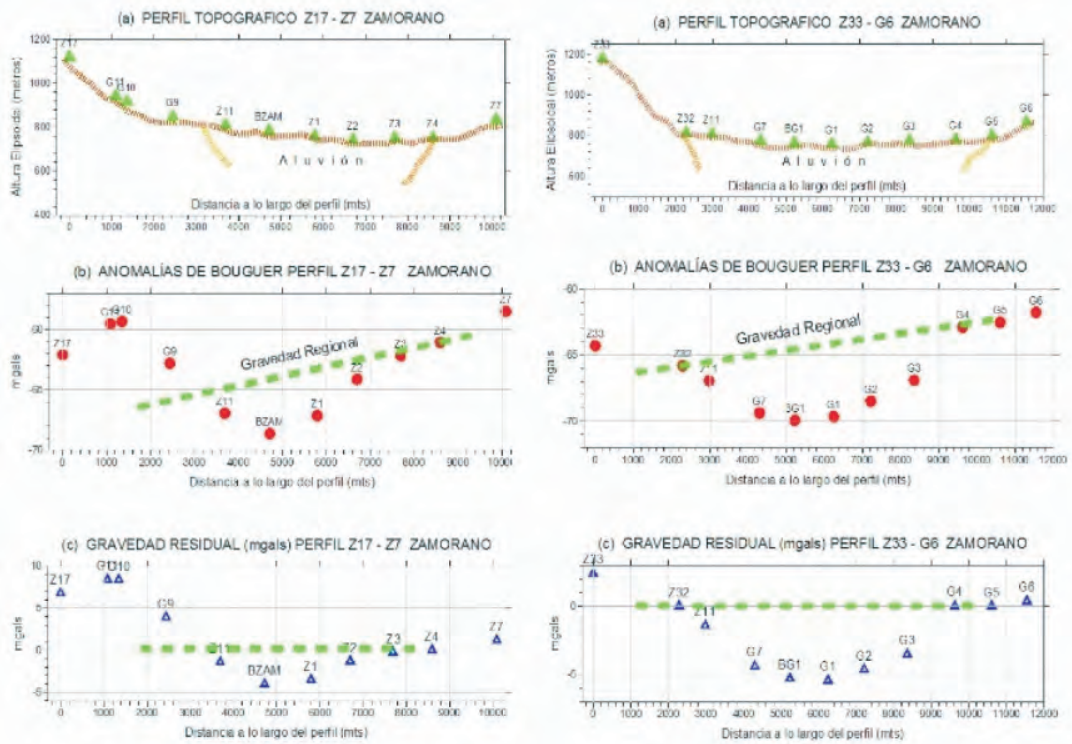

a

C
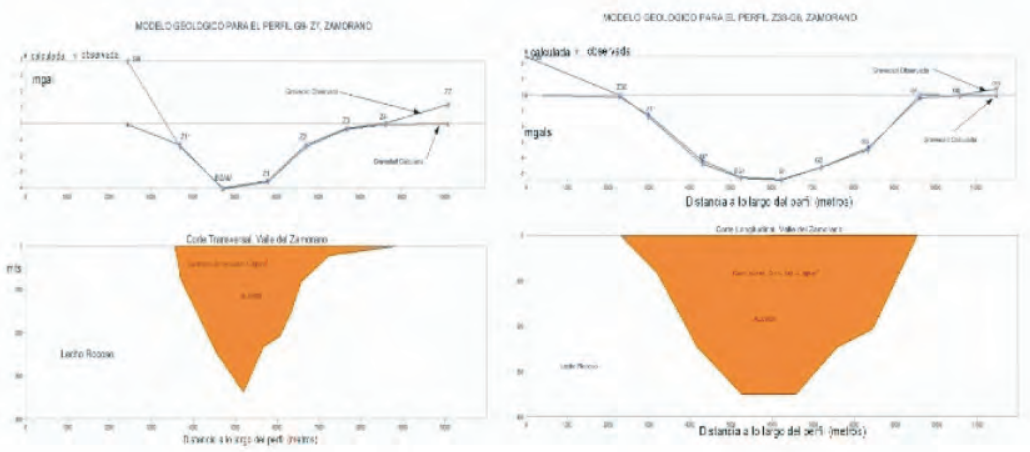

b

d 
Figura 18. (a) Topografía, anomalías de bouguer y gravedad reducida para el perfil transversal Z17-Z7 del Valle del Zamorano. La línea verde indica la definición de gravedad regional para este problema, escogida para que la gravedad residual sea negativa donde haya un contraste de densidad negativo, entre las estaciones Z11 y Z3. (b) Modelo geológico de la cuenca sedimentaria para el perfil Z17-Z7 del Valle del Zamorano. (c) Topografía, anomalías de bouguer y gravedad reducida para el perfil Z33-G6 del Valle del Zamorano. Nuevamente se ha trazado una regional para que los efectos residuales sean atribuidos a los depósitos de aluvión en el valle. En este caso la gravedad residual es negativa entre las estaciones C32 y G4. Los valores máximos de las anomalías residuales están muy cercanos a los -5mgals. (d) Modelo geológico de la cuenca sedimentaria para el perfil C33-G6 del Valle del Zamorano, que corresponde al corte longitudinal del valle, de acuerdo a este modelo la profundidad máxima se observa en el centro del valle y se estima que es alrededor de $350 \mathrm{~m}$.

\section{RECONOCIMIENTOS}

El receptor Trimble5700 que utilizamos (Figura 1) es propiedad del Proyecto "Estudio mediante GPS de la tectónica de Centro América Septentrional", (DeMets, 1998) financiado por la Fundación Nacional de Ciencia (NSF) de los Estados Unidos de América y ejecutado por el Prof. Charles DeMets del Departamento de Geología y Geofísica de la Universidad de Wisconsin en Madison en cooperación con el Departamento de Física de la Universidad Nacional Autónoma de Honduras (UNAH, 2002). El gravímetro empleado GRAVITON EG es propiedad del Prof Basil Tipoff también del Departamento de Geología y Geofísica de la Universidad de Wisconsin, Madison, quien accedió gentilmente al uso del gravímetro. Reconocemos la valiosa asistencia del Lic. Manuel Rodríguez, quien fue el responsable del traslado del equipo desde los E.E.U.U., proporcionando además valiosa información bibliográfica. Este trabajo fue realizado gracias a la Beca Básica de Investigación, otorgada por la Dirección de Investigación Científica (D.I.C.U.) de la Universidad Nacional Autónoma de Honduras (U.N.A.H.)

\section{BIBLIOGRAFIA}

Al-Zadjali, Ibrahim and D.J. Stierman, (1996). Interpretation of Gravity from the Comayagua Graben, Honduras; Abstracts with Programs, North-Central Section of the Geological Society of America, Ames, IA, 1996, p. 25.

Aster, R. C., B. Borchers and C. H. Thurber (2005) Parameter Estimation and Inverse Problems. Elsevier Academic Press.

Blewitt, G. (1997) Basics of the GPS Technique: Observations Equations, in Geodetic Applications of GPS, (Swedish Land Survey). 
Bowin, C. O. (1976) Caribbean Gravity Field and Plate Tectonics. Geol. Soc. Amer. Spec. Pap., 169 1-79. Boulder, Col.

Burger, R, A. F. Sheehan and C.H. Jones (2006) Introduction to Applied Geophysics, exploring the shallow subsurface. W. W. Norton \& Company. New York. London

DeMets, C. (1998) A GPS study of the large-scale neotectonics of northern Central America. Project proposal NSF, award no. EAR 9804905.

Estey, L.H. y C.M. Meertens (1999) TEQC: The Multipurpose Toolkit fro GPS/GLONASS data. GPS Solutions (pub. By John Wiley and Sons) Vol 3, No 1 , pp 42-49.

Hammer, S. (1939) Terrain corrections for gravimeter stations. Geophysics V. 4, 184-94.

Heiskanen, W. A. y H. Moritz (1990) Physical Geodesy. Institute of Physical Geodesy. Technical University. Graz, Austria.

Hubbert, M. King (1948) Line-integral method for computing the gravimetric effects of two-dimensional masses. Geophysics, V. 13, 215-25.

IGN (1970), Mapa Geológico de Honduras, Hoja 2758 IG. Secretaría de Comunicaciones, Obras Públicas y Transporte, Instituto Geográfico Nacional, Tegucigalpa, D.C. Honduras.

IGN (1993), Mapa Geológico de Honduras, Hoja 2758 IIG. Secretaría de Comunicaciones, Obras Públicas y Transporte, Instituto Geográfico Nacional, Tegucigalpa, D.C. Honduras.

IGN (1996), Mapa Geológico de Honduras, Hoja 2858 IIIG. Secretaría de Comunicaciones, Obras Públicas y Transporte, Instituto Geográfico Nacional, Tegucigalpa, D.C. Honduras.

LaCoste \& Romberg LLC. (2002) GRAVITON-EG User's manual (revision 1.8) www.lacosteromberg.com

Leaman D. E. [1998]. The gravity terrain correction - practical considerations. Exploration Geophysics 29, 467-471.

Li, X. y H. J. Götze (2001) Ellipsoid, Geoid, Gravity, Geodesy and Geophysics.

Geophysics, Vol 66, No 6, p 1660-1668.

Markey, R.J., (1993), GEOLÓGIA DEL CUADRANGULO DE MOROCELI, FRANCISCO MORAZAN, Open File Report, Instituto Geográfico Nacional, Tegucigalpa, Honduras.

Menke, W. (1989) Geophysical Data Analysis: Discrete Inverse Theory. Academic Press, Inc.

Nacional Ocean Service and National Geodetic Survey (2001). Establishment of Absolute Gravity Stations as part of Hurricane Mitch restoration program. Project Report, Absolute Gravity Party. 
O'Conner, E. A. (1986). Report on a visit to Tegucigalpa, Honduras. Technical Report MP/86/19/R: British Geological Survey, Keyworth, Nottingham, United Kingdom, $16 \mathrm{pp}$.

Rogers, R. D., (2003), Jurassic-Recent tectonic and stratigraphic history of the Chortis block of Honduras and Nicaragua (northern Central America), The University of Texas atAustin, Ph. D. dissertation, $289 p$.

Rogers, R.D. y E.A. O'Conner, (1993), Mapa Geológico de Honduras: Hoja de Tegucigalpa (segunda edición), Instituto Geográfico Nacional, Tegucigalpa, Honduras, escala 1:50,000.

Rudman, Albert J., Robert Ziegler, and Robert F. Blakely (1977). FORTRAN program for generation of Earth tide gravity values. Indiana Geological Survey Ocassional Paper 22.

Schiavone, D. Capolongo and M. Loddo (2007) High Resolution Dems for Near Station Terrain Correction in Gravimetry. International Workshop Innovation in EM, Grav and Mag Methods:a new Perspective for Exploration. Capri, Italy, April 15 - 18, 2007

Seigel, H. O., (1995) A Guide to High Precision Land Gravimeter Surveys in Applied Geophysics. Scintrex Limited,Ontaro.

Tenorio, C. y E. Espinoza (2007). Estudio Gravimétrico del Cuadrángulo de Tegucigalpa, Valle del Zamorano y Valle de San Juan de Flores y Morocelí. (Reporte Interno) Departamento de Física, Universidad Nacional Autónoma de Honduras.

Talwani, M., J. W. Worzel, and M. Landsman. (1959) Rapad gravity computations for two-dimensional bodies with application to the Mendocino submarine fracture zone. Journal of Geophysical Research

Telford, W. M., L. P. Geldart, R. E. Sheriff and D. A. Keys (1987) Applied Geophysics. Cambridge University Press.

Weyl, R. (1980) Geology of Central America. Second Edition. Gebrüder Borntraeger. Williams, H. yA. R. Mc Birney (1969). Volcanic History of Honduras. Univ. Calf. Publ. Geol. Sci. , V85, 101 pp.

Zuniga, M. (1975) Gravity and Magnetic Survey of the Sula Valley, Honduras, Central America. PhD. Thesis Dissertation . University of Texas atAustin. 